$\underline{\text { Preprint typeset in JHEP style - HYPER VERSION }}$

Preprint TTK-11-01

February 4, 2011

\title{
B polarization of cosmic background radiation from second-order scattering sources
}

\author{
M. Beneke, C. Fidler and K. Klingmüller \\ Institut für Theoretische Teilchenphysik und Kosmologie \\ RWTH Aachen University \\ D - 52056 Aachen, Germany
}

\begin{abstract}
B-mode polarization of the cosmic background radiation is induced from purely scalar primordial sources at second order in perturbations of the homogeneous, isotropic universe. We calculate the B-mode angular power spectrum $C_{l}^{B B}$ sourced by the secondorder scattering term in the full second-order Boltzmann equations for the polarized radiation phase-space density, which have recently become available. We find that at $l \approx 200$ the second-order effect is comparable to the first-order effect for a tensor-to-scalar ratio of $r=10^{-6}$, and to about $2 \cdot 10^{-4}$ at $l \approx 1000$. It is always negligible relative to the weak-lensing induced contribution.

KEYWORDs: Cosmic background radiation, polarization.
\end{abstract}




\section{Contents}

1. Introduction 1

2. Second-order scattering sources of CBR polarization 3

2.1 The B-mode angular power spectrum 3

2.2 Second-order equations

3. Solving the second-order equations 8

3.1 Line-of-sight integration 9

3.2 Solution using Green functions 10

3.3 Second-order power spectra 11

3.4 Non-Gaussianity

4. Numerical evaluation 13

4.1 First-order solutions

4.2 Initial conditions 14

4.3 Green functions

4.4 Closing relations

4.5 Source terms and integration

4.6 Test in the tight-coupling regime 22

4.7 Numerical stability tests 22

5. Result and conclusion 24

A. Summary of equations 27

B. Derivation of the line-of-sight solution 30

\section{Introduction}

Polarization is expected to play a central role in future studies of the cosmic background radiation. The polarization patterns are usually split into a divergence-like E-mode and a curl-like B-mode [1, 2]. B-mode polarization is a powerful diagnostic for primordial gravitational waves, i.e. tensor fluctuations of the metric, and thereby constrains inflation models directly. While E-mode polarization has already been detected [3, 4] and is being observed with increasing precision [5, 6], a B-mode signal remains elusive. This, together with precise information on the temperature anisotropy spectrum indicates some suppression of primordial tensor versus scalar perturbations, since B-modes are not generated by scalar perturbations. 
The absence B-mode polarization when the primordial fluctuations are purely scalar holds, however, only in linear perturbation theory. If primordial tensor fluctuations are indeed suppressed, B-mode polarization generated from scalar sources in second order may constitute an important background to the search for primordial gravitational waves. While such an effect would naturally be expected to be relevant at tensor-to-scalar ratios of order $10^{-5}$, which is the size of perturbations in the microwave background, only a full secondorder calculation can tell whether there are no enhancements. In this paper we compute a new second-order effect that contributes to B-mode polarization.

Several second-order sources of B-mode polarization have already been calculated in various approximations. The most important is the weak-lensing effect, reviewed in Ref. [7], which converts E-mode polarization to B-mode polarization as the photons travel through the inhomogeneous universe [8]. Weak lensing becomes large at small scales, and at large values of the perturbation wave-vector $\boldsymbol{k}$ the perturbation series breaks down. The usual treatment of weak lensing therefore avoids perturbation theory by considering the small deflection angles of the photon trajectories. Another effect that has been estimated is B-mode polarization from gravitational time delay [9] and from sources proportional to second-order vector and tensor metric perturbations, which are themselves generated from the product of scalar perturbations [10].

Recently the full second-order Boltzmann equations for the cosmological evolution of the polarized radiation distribution have become available [11, 12], so that in principle it is possible to compute the power spectrum of B-mode polarization in second order generated from primordial scalar sources without approximations. While this is numerically challenging and beyond the scope of the present paper, we focus here on the novel sources of B-mode polarization that appear in the second-order collision term, which have not been estimated before. As in previous investigations of the weak-lensing and second-order metric perturbation effect, we calculate the amount of B-mode polarization from second-order scattering sources in isolation, that is, we set the sources of other effects to zero in the equations. We then compare the magnitude of the collisional effect to those previously known. Investigating the various effects in isolation may be viewed as a first step to solving the full equations, and is probably a good approximation, since the different effects are related to different periods in the evolution of the universe. In particular, the collision sources relevant to the present paper are active mainly during a short period around recombination, and later, during reionization. The reionization contribution will, however, not be considered here.

The outline of the paper is as follows. In Section 2 we define the B-mode angular power spectrum when the radiation spectrum is not black-body. We briefly review the structure of the second-order polarized Boltzmann equations and discuss the terms that define the new collisional sources. A numerical method based on Green functions, which is suited to compute the two-point function that relates to the angular power spectrum $C_{l}^{B B}$ of B-mode temperature fluctuations, is derived in Section 3. In Section 4 we apply this method to calculate the power spectrum and discuss issues related to numerical checks and stability. Our results and conclusions are summarized in Section 5 . Two appendices collect equations and derivations that supplement Sections 2 and 3 . 


\section{Second-order scattering sources of CBR polarization}

In this section we begin by defining the B-mode angular power spectrum and summarize the second-order Boltzmann equations that will be solved subsequently.

\subsection{The B-mode angular power spectrum}

The cosmic background radiation (CBR) is described by the phase-space density matrix

$$
f_{a b}(\eta, \boldsymbol{x}, \boldsymbol{q})=\delta_{a b} f_{I}^{(0)}(q)+f_{a b}^{(1)}(\eta, \boldsymbol{x}, \boldsymbol{q})+f_{a b}^{(2)}(\eta, \boldsymbol{x}, \boldsymbol{q})+\ldots,
$$

which we expand around the unpolarized, homogeneous black-body background distribution $f_{I}^{(0)}(q)$. Expressed in terms of co-moving phase-space momenta $\boldsymbol{q}=q \boldsymbol{n}, f_{I}^{(0)}(q)$ is time-independent and the black-body temperature $T_{0}$ is the temperature of the CBR today. The indices $a, b= \pm$ refer to the circular polarization basis and can be exchanged for the Stokes parameters $X=I, V, E, B$ by a linear transformation [11]. We define a matrix of fractional temperature perturbations $\Theta_{a b}(\eta, \boldsymbol{x}, \boldsymbol{q})$ through

$$
f_{a b}(\eta, \boldsymbol{x}, \boldsymbol{q})=\left[\exp \left(\frac{q}{T_{0}(1+\Theta(\eta, \boldsymbol{x}, \boldsymbol{q}))}\right)-1\right]_{a b}^{-1},
$$

where the "one" in square parenthesis and in the argument of the exponential function must be interpreted as the $2 \times 2$ unit matrix, and we expand $\Theta(\eta, \boldsymbol{x}, \boldsymbol{q})=\Theta^{(1)}(\eta, \boldsymbol{x}, \boldsymbol{n})+$ $\Theta^{(2)}(\eta, \boldsymbol{x}, \boldsymbol{q})+\ldots$ The term "temperature perturbation" is slightly misleading, since in general $f_{a b}(\eta, \boldsymbol{x}, \boldsymbol{q})$ is not a Bose-Einstein distribution when perturbations are included, and hence $\Theta(\eta, \boldsymbol{x}, \boldsymbol{q})$ is not independent of the photon energy $q$. However, as is well-known, the first-order perturbation $\Theta^{(1)}(\eta, \boldsymbol{x}, \boldsymbol{n})$ is independent of $q$, and the spectrum remains black-body at first order with a position- and direction-dependent temperature. At second order, however, distortions of the black-body spectrum are expected.

We wish to consider observables independent of $q$, which may be interpreted as temperature perturbations. To this end we define the radiation energy density normalized to the unperturbed energy density,

$$
\Delta_{a b}(\eta, \boldsymbol{x}, \boldsymbol{n})=\frac{\int d q q^{3} f_{a b}(\eta, \boldsymbol{x}, q \boldsymbol{n})}{\int d q q^{3} f_{I}^{(0)}(q)} .
$$

If the photons were unpolarized and assumed a black-body spectrum with temperature $T(\eta, \boldsymbol{x}, \boldsymbol{n})$, then $\Delta(\eta, \boldsymbol{x}, \boldsymbol{n})$ would be related to the temperature perturbation by

$$
\Delta(\eta, \boldsymbol{x}, \boldsymbol{n})=\left[\frac{T(\eta, \boldsymbol{x}, \boldsymbol{n})}{T_{0}}\right]^{4}=(1+\Theta(\eta, \boldsymbol{x}, \boldsymbol{n}))^{4} .
$$

In general we obtain the matrix identities

$$
\begin{aligned}
& \Delta^{(1)}(\eta, \boldsymbol{x}, \boldsymbol{n})=4 \Theta^{(1)}(\eta, \boldsymbol{x}, \boldsymbol{n}), \\
& \Delta^{(2)}(\eta, \boldsymbol{x}, \boldsymbol{n})=4 \bar{\Theta}^{(2)}(\eta, \boldsymbol{x}, \boldsymbol{n})+6\left[\Theta^{(1)}(\eta, \boldsymbol{x}, \boldsymbol{n})\right]^{2},
\end{aligned}
$$


among the perturbation coefficients, where

$$
\bar{\Theta}^{(2)}(\eta, \boldsymbol{x}, \boldsymbol{n})=\frac{\int d q q^{3} f_{I}^{(0)}(q)\left[\Theta^{(2)}(\eta, \boldsymbol{x}, q \boldsymbol{n})+\frac{1}{4} q \frac{\partial}{\partial q} \Theta^{(2)}(\eta, \boldsymbol{x}, q \boldsymbol{n})\right]}{\int d q q^{3} f_{I}^{(0)}(q)} .
$$

The quantity $\bar{\Theta}^{(2)}$ obviously equals $\Theta^{(2)}$ when the latter is $q$ independent. Otherwise it represents the temperature perturbation of a black-body distribution with the same energy density as the actual radiation distribution $f(\eta, \boldsymbol{x}, \boldsymbol{q})$.

We define the multipole expansion coefficients of the fractional temperature perturbation here and today as usual by

$$
a_{l m}=a_{l m}^{(1)}+a_{l m}^{(2)}+\ldots=\int d \Omega(\boldsymbol{n}) Y_{l m}^{s *}(\boldsymbol{n})\left[\Theta^{(1)}\left(\eta_{0}, \boldsymbol{x}_{0}, \boldsymbol{n}\right)+\bar{\Theta}^{(2)}\left(\eta_{0}, \boldsymbol{x}_{0}, \boldsymbol{n}\right)+\ldots\right],
$$

noting the presence of the equivalent black-body temperature perturbation and the $2 \times 2$ matrix nature of $a_{l m}$. The spin of the spin-weighted spherical harmonic, $Y_{l m}^{s}(\boldsymbol{n})$, is $s=0$ for the diagonal,++-- components, $s=+2$ for $a b=+-$, and $s=-2$ for -+ . In the circular polarization basis B-mode polarization is given by $i / 2$ times the difference of the +- and -+ components of the $2 \times 2$ density matrix, hence we define

$$
a_{B, l m}=\frac{i}{2}\left(a_{+-, l m}-a_{-+, l m}\right) .
$$

The absence of B-mode polarization in first order implies that the +- and -+ components of $f_{a b}^{(1)}$ and $\Theta_{a b}^{(1)}$ as well as $\left[\Theta^{(1)}\right]_{a b}^{2}$ are equal, so $a_{B, l m}^{(1)}=0$. At second order

$$
\begin{aligned}
a_{B, l m}^{(2)} & =\frac{1}{4} \int d \Omega(\boldsymbol{n}) \frac{i}{2}\left[Y_{l m}^{+2 *}(\boldsymbol{n}) \Delta_{+-}^{(2)}\left(\eta_{0}, \boldsymbol{x}_{0}, \boldsymbol{n}\right)-Y_{l m}^{-2 *}(\boldsymbol{n}) \Delta_{-+}^{(2)}\left(\eta_{0}, \boldsymbol{x}_{0}, \boldsymbol{n}\right)\right] \\
& =\frac{1}{4} \int \frac{d^{3} \boldsymbol{k}}{(2 \pi)^{3}} e^{i \boldsymbol{k} \cdot \boldsymbol{x}_{0}}(-i)^{l} \sqrt{\frac{4 \pi}{2 l+1}} \Delta_{B, l m}^{(2)}\left(\eta_{0}, \boldsymbol{k}\right),
\end{aligned}
$$

where in the last line we introduced the Fourier modes and multipole coefficients of $\Delta_{B}^{(2)}\left(\eta_{0}, \boldsymbol{x}_{0}, \boldsymbol{n}\right)$.

Our aim is to compute the B-mode angular power spectrum $C_{l}^{B B}$ given by the statistical average

$$
\left\langle a_{B, l m} a_{B, l^{\prime} m^{\prime}}^{*}\right\rangle=\delta_{l l^{\prime}} \delta_{m m^{\prime}} C_{l}^{B B}
$$

when the perturbations of the FRW background at first order are purely scalar. Using Eq. (2.9) this average can be expressed in terms of

$$
\left\langle\Delta_{B, l m}^{(2)}\left(\eta_{0}, \boldsymbol{k}\right) \Delta_{B, l^{\prime} m^{\prime}}^{(2) *}\left(\eta_{0}, \boldsymbol{k}^{\prime}\right)\right\rangle=(2 \pi)^{3} \delta^{(3)}\left(\boldsymbol{k}-\boldsymbol{k}^{\prime}\right) P_{l l^{\prime}, m m^{\prime}}^{\Delta_{B}}(k, \hat{\boldsymbol{k}})
$$

in the form

$$
\left\langle a_{B, l m} a_{B, l^{\prime} m^{\prime}}^{*}\right\rangle=\frac{1}{16}(-i)^{l} i^{l^{\prime}} \frac{4 \pi}{\sqrt{(2 l+1)\left(2 l^{\prime}+1\right)}} \int \frac{d^{3} \boldsymbol{k}}{(2 \pi)^{3}} P_{l l^{\prime}, m m^{\prime}}^{\Delta_{B}}(k, \hat{\boldsymbol{k}}) .
$$

The power spectrum of $\Delta_{B}$ defined here depends on the direction $\hat{\boldsymbol{k}}$ of the mode vector, since it refers to the fixed coordinate system of an observer and helicity $m$ defined with 
respect to the three-axis of this system. When the helicity axis is chosen to be $\hat{\boldsymbol{k}}$ we obtain the simpler expression

$$
\left\langle\Delta_{B, l m}^{(2)}\left(\eta_{0}, \boldsymbol{k}\right) \Delta_{B, l^{\prime} m^{\prime}}^{(2) *}\left(\eta_{0}, \boldsymbol{k}^{\prime}\right)\right\rangle_{\mid \hat{\boldsymbol{k}} \text { axis }}=\delta_{m m^{\prime}}(2 \pi)^{3} \delta^{(3)}\left(\boldsymbol{k}-\boldsymbol{k}^{\prime}\right) P_{l l^{\prime}, m}^{\Delta_{B}}(k)
$$

where the form of the right hand side of the equation follows from statistical isotropy and homogeneity and explicitly from the results of Section 3. In particular, the power spectrum $P_{l l^{\prime}, m}^{\Delta B}(k)$ depends only on the magnitude $k=|\boldsymbol{k}|$. It is this power spectrum that will be computed later on for $l^{\prime}=l$. The two power spectra are related through the transformation A.5) of the B-mode multipoles under rotations, which gives

$$
P_{l l^{\prime}, m m^{\prime}}^{\Delta_{B}}(k, \hat{\boldsymbol{k}})=\frac{4 \pi}{\sqrt{(2 l+1)\left(2 l^{\prime}+1\right)}} \sum_{\tilde{m}} Y_{l m}^{-\tilde{m}}(\hat{\boldsymbol{k}}) Y_{l^{\prime} m^{\prime}}^{-\tilde{\tilde{k}} *}(\hat{\boldsymbol{k}}) P_{l l^{\prime}, \tilde{m}}^{\Delta_{B}}(k) .
$$

Plugging this into Eq. (2.12) and using the orthogonality of the spin-weighted spherical harmonics, we obtain the familiar result

$$
C_{l}^{B B}=\frac{1}{16} \frac{2}{\pi} \sum_{m= \pm 1, \pm 2} \int_{0}^{\infty} d k k^{2} \frac{P_{l l, m}^{\Delta_{B}}(k)}{(2 l+1)^{2}}
$$

The sum is restricted to values $|m| \leq 2$, since the equations for $\Delta_{X, l m}^{(2)}(\boldsymbol{k})$ in the frame where $\hat{\boldsymbol{k}}$ coincides with the helicity axis do not contain source terms when $|m|>2$. The sum does not include $m=0$ since there are no scalar mode contributions to B-mode polarization.

\subsection{Second-order equations}

In Ref. [11] we derived the second-order Boltzmann equations for the photon phase-space densities $f_{X, l m}(\eta, \boldsymbol{k}, q)$. We now explain the structure of these equations and discuss the approximations we apply in this paper to isolate the second-order scattering sources. We are interested in the energy integrated distribution functions defined by

$$
\Delta_{X, l m}^{(n)}(\eta, \boldsymbol{k})=\frac{\int d q q^{3} f_{X, l m}^{(n)}(\eta, \boldsymbol{k}, q)}{\int d q q^{3} f_{I}^{(0)}(q)}
$$

in particular in $\Delta_{B, l m}^{(2)}(\eta, \boldsymbol{k})$. The equation for second-order B-mode polarization when there are no first-order vector and tensor perturbations, hence $\Delta_{B, l m}^{(1)}(\eta, \boldsymbol{k})=0$, is given by

$$
\begin{aligned}
& \frac{\partial}{\partial \eta} \Delta_{B, l m}^{(2)}(\boldsymbol{k})+\sum_{ \pm}(\mp i) \Delta_{B,(l \pm 1) m_{1}}^{(2)}(\boldsymbol{k}) k^{\left[m_{2}\right]} D_{m_{1} m}^{ \pm, l}+i \Delta_{E, l m_{1}}^{(2)}(\boldsymbol{k}) k^{\left[m_{2}\right]} D_{m_{1} m}^{0, l} \\
& +i k_{1}^{\left[m_{2}\right]}\left(A^{(1)}-D^{(1)}\right)\left(\boldsymbol{k}_{1}\right) \Delta_{E, l m_{1}}^{(1)}\left(\boldsymbol{k}_{2}\right) K_{m_{1} m}^{0, l} \\
& +\left(i k_{2}^{\left[m_{2}\right]}\left(A^{(1)}-D^{(1)}\right)\left(\boldsymbol{k}_{1}\right)+4 i k_{1}^{\left[m_{2}\right]} A^{(1)}\left(\boldsymbol{k}_{1}\right)\right) \Delta_{E, l m_{1}}^{(1)}\left(\boldsymbol{k}_{2}\right) D_{m_{1} m}^{0, l} \\
& =|\dot{\kappa}|\left\{-\Delta_{B, l m}^{(2)}(\boldsymbol{k})+v_{e,\left[m_{2}\right]}^{(1)}\left(\boldsymbol{k}_{1}\right) \Delta_{E, l m_{1}}^{(1)}\left(\boldsymbol{k}_{2}\right) D_{m_{1} m}^{0, l}\right. \\
& \left.-\delta_{l 2} \frac{\sqrt{6}}{5} v_{e,\left[m_{2}\right]}^{(1)}\left(\boldsymbol{k}_{1}\right)\left(\Delta_{I, 2 m_{1}}^{(1)}-\sqrt{6} \Delta_{E, 2 m_{1}}^{(1)}\right)\left(\boldsymbol{k}_{2}\right) D_{m_{1} m}^{0,2}\right\} \text {. }
\end{aligned}
$$


Some comments on notation are in order. The equations above and below are given in conformal Newtonian gauge for phase-space densities defined in an inertial frame locally at rest and aligned with respect to the general coordinate system. $A^{(1)}$ and $D^{(1)}$ denote the first-order scalar metric perturbations, $v_{e,[m]}$ the velocity field of the baryon-electron fluid, and $|\dot{\kappa}|$ is proportional to the collision rate of Thomson scattering. The coupling coefficients $D_{m_{1} m}^{0, l}$ etc. are summarized in Eq. (A.10) and Ref. [11]. Products of firstorder perturbations involve two different mode vectors $\boldsymbol{k}_{1}$ and $\boldsymbol{k}_{2}=\boldsymbol{k}-\boldsymbol{k}_{1}$ and it is understood that a convolution integral over $\boldsymbol{k}_{1}$ is performed. Similarly, for given $m$ we imply $m_{2}=m-m_{1}$ and a sum over $m_{1}$. Indices in square brackets refer to helicity rather than Cartesian vector components with $k^{[0]}=i k_{3}$. We refer to Ref. [11] for further notational details.

Eq. (2.17) and the corresponding equations for the radiation intensity and E-mode polarization contain a number of different effects:

- In addition to the conformal time derivative the first line contains the effect of free streaming of radiation perturbations in the unperturbed background. This term converts vector and tensor E-mode perturbations into the corresponding B-mode perturbations and further excites higher multipoles from the $l=0,1,2$ ones after radiation ceases to be tightly coupled to the baryons. These terms must obviously be included in a calculation of the angular power spectrum observed today.

- The remaining two lines before the equality sign are the weak lensing and time delay contributions, which represent the effect of space-time inhomogeneity on the photon perturbations as they travel through the universe. This converts E-mode polarization into B-mode polarization even in the absence of vector and tensor perturbations and is known to generate significant B-mode polarization at large $l$ [8]. We drop these known contributions, since we are interested in the effect of the collision source terms.

- The equation for $\Delta_{I, l m}^{(2)}(\boldsymbol{k})$ contains second-order metric source terms involving products of first-order perturbations such as $A^{(1)}\left(\boldsymbol{k}_{1}\right) D^{(1)}\left(\boldsymbol{k}_{2}\right)$ and second-order perturbations $A^{(2)}(\boldsymbol{k}), \dot{B}_{[m]}^{(2)}(\boldsymbol{k})$ and $\dot{E}_{[m]}^{(2)}(\boldsymbol{k})$. The amount of B-mode polarization generated from some of these terms has been estimated in Ref. [10]. Specifically, the vector and tensor perturbations $B_{[m]}^{(2)}(\boldsymbol{k})$ and $E_{[m]}^{(2)}(\boldsymbol{k})$ induced at second-order from purely scalar perturbations have been evaluated and inserted into the first-order equations for free streaming to obtain the amount of B-mode polarization today. Again, since we are interested in the effect of the new collision source terms, we drop these terms.

- The right-hand side of Eq. (2.17) is the second-order collision term. The first term in curly brackets is a universal relaxation term for all multipoles that in the absence of source terms drives the phase-space distribution to its equilibrium form and hence damps polarization. This term is already present in first order. In addition we find new scattering sources of B-mode polarization from the coupling of first-order scalar intensity and E-mode polarization to perturbations in the electron-baryon bulk velocity. These terms and corresponding new terms in the equations for $\Delta_{I, l m}^{(2)}(\boldsymbol{k})$ 
and $\Delta_{E, l m}^{(2)}(\boldsymbol{k})$ are kept in our numerical calculation. They constitute the scattering sources, whose effect is computed here for the first time. We thus include the complete second-order collision term with one exception. We neglect terms of the form $|\dot{\kappa}|\left[\delta x_{e} / x_{e}\right]^{(1)} \times$ first order perturbations, which arise from the perturbed ionization history.

We thus solve the following system of second-order equations:

$$
\begin{aligned}
\frac{\partial}{\partial \eta} \Delta_{I, l m}^{(2)}(\boldsymbol{k})+ & \sum_{ \pm}(\mp i) \Delta_{I,(l \pm 1) m_{1}}^{(2)}(\boldsymbol{k}) k^{\left[m_{2}\right]} C_{m_{1} m}^{ \pm, l} \\
=|\dot{\kappa}|\{ & -\Delta_{I, l m}^{(2)}(\boldsymbol{k})+\delta_{l 0} \Delta_{I, 00}^{(2)}(\boldsymbol{k})+4 \delta_{l 1} v_{e,[m]}^{(2)}(\boldsymbol{k})+\delta_{l 2} \frac{1}{10}\left(\Delta_{I, 2 m}^{(2)}(\boldsymbol{k})-\sqrt{6} \Delta_{E, 2 m}^{(2)}(\boldsymbol{k})\right) \\
& \left.+A^{(1)}\left(\boldsymbol{k}_{1}\right)+\delta_{b}^{(1)}\left(\boldsymbol{k}_{1}\right)\right)\left(-\Delta_{I, l m}^{(1)}\left(\boldsymbol{k}_{2}\right)+\delta_{l 0} \Delta_{I, 00}^{(1)}\left(\boldsymbol{k}_{2}\right)+4 \delta_{l 1} v_{e,[m]}^{(1)}\left(\boldsymbol{k}_{2}\right)\right. \\
& \left.+\delta_{l 2} \frac{1}{10}\left(\Delta_{I, 2 m}^{(1)}-\sqrt{6} \Delta_{E, 2 m}^{(1)}\right)\left(\boldsymbol{k}_{2}\right)\right) \\
+ & \sum_{ \pm}(\mp 1) v_{e,\left[m_{2}\right]}^{(1)}\left(\boldsymbol{k}_{1}\right) \Delta_{I,(l \pm 1) m_{1}}^{(1)}\left(\boldsymbol{k}_{2}\right) C_{m_{1} m}^{ \pm, l} \\
+ & \delta_{l 0} v_{e,\left[m_{2}\right]}^{(1)}\left(\boldsymbol{k}_{1}\right)\left(2 \Delta_{I, 1 m_{1}}^{(1)}-4 v_{e,\left[m_{1}\right]}^{(1)}\right)\left(\boldsymbol{k}_{2}\right) C_{m_{1} m}^{+, 0} \\
+ & 3 \delta_{l 1} v_{e,\left[m_{2}\right]}^{(1)}\left(\boldsymbol{k}_{1}\right) \Delta_{I, 0 m_{1}}^{(1)}\left(\boldsymbol{k}_{2}\right) C_{m_{1} m}^{-, 1} \\
+ & \delta_{l 2} v_{e,\left[m_{2}\right]}^{(1)}\left(\boldsymbol{k}_{1}\right)\left(7 v_{e,\left[m_{1}\right]}^{(1)}\left(\boldsymbol{k}_{2}\right)-\frac{1}{2} \Delta_{I, 1 m_{1}}^{(1)}\left(\boldsymbol{k}_{2}\right)\right) C_{m_{1} m}^{-, 2} \\
+ & \left.\frac{1}{2} \delta_{l 3} v_{e,\left[m_{2}\right]}^{(1)}\left(\boldsymbol{k}_{1}\right)\left(\Delta_{I, 2 m_{1}}^{(1)}-\sqrt{6} \Delta_{E, 2 m_{1}}^{(1)}\right)\left(\boldsymbol{k}_{2}\right) C_{m_{1} m}^{-, 3}\right\}
\end{aligned}
$$

$$
\begin{aligned}
\frac{\partial}{\partial \eta} \Delta_{E, l m}^{(2)}(\boldsymbol{k}) & +\sum_{ \pm}(\mp i) \Delta_{E,(l \pm 1) m_{1}}^{(2)}(\boldsymbol{k}) k^{\left[m_{2}\right]} D_{m_{1} m}^{ \pm, l}-i \Delta_{B, l m_{1}}^{(2)}(\boldsymbol{k}) k^{\left[m_{2}\right]} D_{m_{1} m}^{0, l} \\
=|\dot{\kappa}|\{ & -\Delta_{E, l m}^{(2)}(\boldsymbol{k})-\delta_{l 2} \frac{\sqrt{6}}{10}\left(\Delta_{I, 2 m}^{(2)}(\boldsymbol{k})-\sqrt{6} \Delta_{E, 2 m}^{(2)}(\boldsymbol{k})\right) \\
& +\left(A^{(1)}\left(\boldsymbol{k}_{1}\right)+\delta_{b}^{(1)}\left(\boldsymbol{k}_{1}\right)\right)\left(-\Delta_{E, l m}^{(1)}\left(\boldsymbol{k}_{2}\right)-\delta_{l 2} \frac{\sqrt{6}}{10}\left(\Delta_{I, 2 m}^{(1)}-\sqrt{6} \Delta_{E, 2 m}^{(1)}\right)\left(\boldsymbol{k}_{2}\right)\right) \\
& +\sum_{ \pm}(\mp 1) v_{e,\left[m_{2}\right]}^{(1)}\left(\boldsymbol{k}_{1}\right) \Delta_{E,(l \pm 1) m_{1}}^{(1)}\left(\boldsymbol{k}_{2}\right) D_{m_{1} m}^{ \pm, l} \\
& +\delta_{l 2} \frac{\sqrt{6}}{2} v_{e,\left[m_{2}\right]}^{(1)}\left(\boldsymbol{k}_{1}\right)\left(\Delta_{I, 1 m_{1}}^{(1)}\left(\boldsymbol{k}_{2}\right)-2 v_{e,\left[m_{1}\right]}^{(1)}\left(\boldsymbol{k}_{2}\right)\right) C_{m_{1} m}^{-, 2} \\
& \left.-\delta_{l 3} \frac{\sqrt{6}}{2} v_{e,\left[m_{2}\right]}^{(1)}\left(\boldsymbol{k}_{1}\right)\left(\Delta_{I, 2 m_{1}}^{(1)}-\sqrt{6} \Delta_{E, 2 m_{1}}^{(1)}\right)\left(\boldsymbol{k}_{2}\right) D_{m_{1} m}^{-, 3}\right\}
\end{aligned}
$$




$$
\begin{gathered}
\frac{\partial}{\partial \eta} \Delta_{B, l m}^{(2)}(\boldsymbol{k})+\sum_{ \pm}(\mp i) \Delta_{B,(l \pm 1) m_{1}}^{(2)}(\boldsymbol{k}) k^{\left[m_{2}\right]} D_{m_{1} m}^{ \pm, l}+i \Delta_{E, l m_{1}}^{(2)}(\boldsymbol{k}) k^{\left[m_{2}\right]} D_{m_{1} m}^{0, l} \\
=|\dot{\kappa}|\left\{-\Delta_{B, l m}^{(2)}(\boldsymbol{k})+v_{e,\left[m_{2}\right]}^{(1)}\left(\boldsymbol{k}_{1}\right) \Delta_{E, l m_{1}}^{(1)}\left(\boldsymbol{k}_{2}\right) D_{m_{1} m}^{0, l}\right. \\
\left.\quad-\delta_{l 2} \frac{\sqrt{6}}{5} v_{e,\left[m_{2}\right]}^{(1)}\left(\boldsymbol{k}_{1}\right)\left(\Delta_{I, 2 m_{1}}^{(1)}-\sqrt{6} \Delta_{E, 2 m_{1}}^{(1)}\right)\left(\boldsymbol{k}_{2}\right) D_{m_{1} m}^{0,2}\right\} \\
\left(\frac{\partial}{\partial \eta}+H_{C}\right) v_{e,[m]}^{(2)}(\boldsymbol{k})=-\frac{|\dot{\kappa}|}{4 R}\left\{4 v_{e,[m]}^{(2)}(\boldsymbol{k})-\Delta_{I, 1 m}^{(2)}(\boldsymbol{k})\right. \\
+A^{(1)}\left(\boldsymbol{k}_{1}\right)\left(4 v_{e,[m]}^{(1)}\left(\boldsymbol{k}_{2}\right)-\Delta_{I, 1 m}^{(1)}\left(\boldsymbol{k}_{2}\right)\right)-v_{e,\left[m_{2}\right]}^{(1)}\left(\boldsymbol{k}_{1}\right) \Delta_{I, 2 m_{1}}^{(1)}\left(\boldsymbol{k}_{2}\right) C_{m_{1} m}^{+, 1} \\
\left.+4 v_{e,[m]}^{(1)}\left(\boldsymbol{k}_{1}\right) \Delta_{I, 00}^{(1)}\left(\boldsymbol{k}_{2}\right)\right\} .
\end{gathered}
$$

with $R=3 \bar{\rho}_{b} /\left(4 \bar{\rho}_{\gamma}\right)$. The equation for $v_{e,[m]}^{(2)}(\boldsymbol{k})$ must be included, since this quantity appears in the collision term of the intensity perturbation equation (2.18), and we applied the same approximations to this equation as for the other three. That is, we neglect the second-order metric perturbation $B_{[m]}^{(2)}$, and for consistency also products of first-order metric perturbations (since $B_{[m]}^{(2)}$ is itself sourced by a product of such terms). The complete equation for $v_{e,[m]}^{(2)}$ and the baryon density perturbation $\delta_{b}^{(2)}=\left[\delta \rho_{b} / \bar{\rho}_{b}\right]^{(2)}$ and the precise definition of these quantities is provided in Appendix A. Eqs. (2.18) to (2.21) form a closed system together with the equations for the first-order perturbations also summarized in the Appendix, which we solve in the following. We note that the approximations made are systematic in the sense that in the absence of collision terms $(|\dot{\kappa}| \rightarrow 0)$ all second-order perturbations vanish; hence we exclusively focus on collisional effects as intended.

\section{Solving the second-order equations}

For convenience, we introduce a compact notation summarizing the photon equations Eq. 2.18) to Eq. (2.20):

$$
\dot{\Delta}_{n}^{(2)}+k C_{n m} \Delta_{m}^{(2)}=-|\dot{\kappa}|\left(\Delta_{n}^{(2)}-\varsigma_{n m} \Delta_{m}^{(2)}-S_{n}\right) .
$$

The indices are multi-indices, $n=\left(X_{n}, l_{n} m_{n}\right)$, where $X=I, E, B, v_{e}$ distinguishes between the photon modes and the baryon velocity; $l, m$ are the multipole indices. Repeated multiindices are summed over $X, l$, and $m ; \boldsymbol{k}$ has been aligned with the three-direction, $\boldsymbol{k}=k \boldsymbol{e}_{3}$. The dependence on $k$ of $\Delta_{n}, S_{n}$, and the Green functions $G_{n m}$ introduced below will not be made explicit. Note that Eq. (3.1) does not represent the electron equation Eq. (2.21), i.e., it is only valid for $n \neq\left(v_{e}, 1 m\right)$.

The matrix $C_{n m}$ describes the free-streaming coupling of each photon multipole moment to its neighbours with $l \pm 1$, which leads to a gradual excitation of the initially small large- $l$ moments. The conformal time it takes for an excitation to propagate from multipole 
moment $l$ to moment $l \pm \Delta l$ is $\eta \approx \Delta l / k$. Free streaming also accounts for the conversion between E- and B-mode polarization for vector and tensor modes, which is the only possible source for B-mode polarization at first order.

The first part of the scattering term, $-|\dot{\kappa}| \Delta_{n}^{(2)}$, is responsible for the tight-coupling suppression. At early times the scattering rate $|\dot{\kappa}|$ is large, so that a non-vanishing moment $\Delta_{n}^{(2)}$ induces a large gradient driving it to zero as long as the first part of the scattering term is not cancelled by the remaining term $\varsigma_{n m} \Delta_{m}^{(2)}+S_{n}$. Since the coupling $\varsigma_{n m}$ vanishes for high multipole moments, only the monopole and dipole are not suppressed by the tight coupling of photons and baryons. At second order the quadrupole $\Delta_{I, 2 m}$ is also present due to a cancellation with $S_{n}$ [11, 12, 13], but there is no polarization in tight coupling [11].

The source $S_{n}$ contains the convolutions of first-order perturbations. Other than in the first-order equations, where there are only source terms for low multipole moments, in the second order source $S_{n}$ there are contributions for all multipoles. Moreover, there is a source term for B-mode polarization which is induced by first-order quadrupoles, including the large intensity quadrupole, cf. Eq. (2.20),

$$
S_{B, l m}=\cdots-\delta_{l 2} \frac{\sqrt{6}}{5} v_{e,\left[m_{2}\right]}^{(1)}\left(\boldsymbol{k}_{1}\right) \Delta_{I, 2 m_{1}}^{(1)}\left(\boldsymbol{k}_{2}\right) D_{m_{1} m}^{0,2} \cdots .
$$

Such a direct source term for B polarization does not exist at first order, where B-mode polarization is only generated from free streaming of vector and tensor E-mode polarization.

\subsection{Line-of-sight integration}

At first order the line-of-sight integration is used to great success [14, 15]. It is the solution of

$$
\dot{\Delta}_{n}+k C_{n m} \Delta_{m}=-|\dot{\kappa}| \Delta_{n}+\rho_{n}
$$

where $C_{n m}$ are the free-streaming coefficients introduced in Eq. (3.1) and $\rho_{n}$ includes all source terms. The solution to this equation is given by

$$
\Delta_{n}\left(\eta_{0}\right)=\int_{0}^{\eta_{0}} d \eta e^{-\kappa(\eta)} j_{n m}\left(k\left(\eta_{0}-\eta\right)\right) \rho_{m}(\eta)
$$

where the functions $j_{n m}$ are combinations of spherical Bessel functions and Clebsch-Gordan coefficients,

$$
\begin{aligned}
j_{n m}(x)= & \sum_{l_{1}} i^{l_{n}-l_{1}-l_{m}} \frac{\left(2 l_{n}+1\right)\left(2 l_{1}+1\right)}{2 l_{m}+1} j_{l_{1}}(x) H_{X_{n} X_{m}}^{*}\left(l_{n}-l_{1}-l_{m}\right) \\
& \times\left(\begin{array}{ccc}
l_{n} & l_{1} & l_{m} \\
m_{n} & 0 & m_{m}
\end{array}\right)\left(\begin{array}{ccc}
l_{n} & l_{1} & l_{m} \\
F_{X_{n}} & 0 & F_{X_{m}}
\end{array}\right) .
\end{aligned}
$$

The matrix $H_{X X^{\prime}}$ is responsible for the mixing between $E$ and $B$ polarization in free streaming and is given by $H_{X X^{\prime}}$ (even) $=\delta_{X X^{\prime}}$ and $H_{X X^{\prime}}($ odd $)=\delta_{X I} \delta_{X^{\prime} I}+i \delta_{X E} \delta_{X^{\prime} B}-$ $i \delta_{X B} \delta_{X^{\prime} E}$ [1]; $F_{X}=0$ when $X=I$ and $F_{X}=-2$ when $X=E, B$. Clebsch-Gordan coefficients are denoted by big round brackets. A derivation of this solution is given in Appendix B. The functions $j_{n m}$ are always real since the product $i^{l_{n}-l_{1}-l_{m}} H_{X_{n} X_{m}}^{*}$ can 
be imaginary only for odd $l_{n}-l_{1}-l_{m}$ and $X_{n}=X_{m}=I$, but in that case the second Clebsch-Gordan coefficient vanishes.

To employ this solution at second order we only need to use the appropriate source terms. In Eq. (3.1) we identify $\rho_{n}=|\dot{\kappa}|\left(S_{n}+\varsigma_{n m} \Delta_{m}^{(2)}\right)$ and we obtain the integral solution

$$
\Delta_{n}^{(2)}\left(\eta_{0}\right)=\int_{0}^{\eta_{0}} d \eta|\dot{\kappa}(\eta)| e^{-\kappa(\eta)} j_{n m}\left(k\left(\eta_{0}-\eta\right)\right)\left(S_{m}+\varsigma_{m p} \Delta_{p}^{(2)}\right)(\eta) .
$$

Despite the second-order term in the integrand, this is a big step towards the computation of second-order quantities today: the coefficient $\varsigma_{m p}$ vanishes for multipole moments $l_{p}>2$ so that we are left with the task of finding solutions for $\Delta_{p}^{(2)}(\eta)$ with $l \leq 2$. In addition, the visibility function $|\dot{\kappa}| e^{-\kappa}$ is non-zero only around recombination and thus it is sufficient to compute these solutions for early times $\eta \lesssim 500 \mathrm{Mpc} / c$. (We quote conformal time in units of $\mathrm{Mpc} / c$, but set the speed of light $c=1$ in equations.)

\subsection{Solution using Green functions}

The Boltzmann equations have to be solved with stochastic initial conditions resulting from inflation. At first order, the linearity of the equations allows to write the solution as product of transfer functions and the primordial fluctuations. The problem reduces to the task of computing the non-stochastic transfer functions. This straightforward separation is no longer possible at second order due to the quadratic convolution terms. However, we can achieve a similar separation by using Green functions.

The second-order photon equation Eq. (3.1) is linear in the second-order quantities,

$$
\dot{\Delta}_{n}^{(2)}(\eta)=A_{n m}(\eta) \Delta_{m}^{(2)}(\eta)+\sigma_{n}(\eta),
$$

where $A_{n m}=|\dot{\kappa}|\left(\varsigma_{n m}-\delta_{n m}\right)-k C_{n m}$ and $\sigma_{n}=|\dot{\kappa}| S_{n}$. The same applies to the electronvelocity equation with $A_{n m}$ and $\sigma_{n}$ according to Eq. (2.21). The solution of such a linear differential equation can be written in terms of Green functions,

$$
\Delta_{n}^{(2)}(\eta)=G_{n m}\left(\eta, \eta_{\mathrm{ini}}\right) \Delta_{m}^{(2)}\left(\eta_{\mathrm{ini}}\right)+\int_{\eta_{\mathrm{ini}}}^{\eta} d \eta^{\prime} G_{n m}\left(\eta, \eta^{\prime}\right) \sigma_{m}\left(\eta^{\prime}\right)
$$

where the Green function $\mathcal{G}_{n m}\left(\eta, \eta^{\prime}\right)=G_{n m}\left(\eta, \eta^{\prime}\right) \theta\left(\eta-\eta^{\prime}\right)$ satisfies

$$
\partial_{\eta} \mathcal{G}_{n m}\left(\eta, \eta^{\prime}\right)=A_{n p}(\eta) \mathcal{G}_{p m}\left(\eta, \eta^{\prime}\right)+\delta_{n m} \delta\left(\eta-\eta^{\prime}\right)
$$

In this differential equation there are no stochastic quantities, thus we can compute the Green function $G_{n m}\left(\eta, \eta^{\prime}\right)$ by solving the equation

$$
\partial_{\eta} G_{n m}\left(\eta, \eta^{\prime}\right)=A_{n p}(\eta) G_{p m}\left(\eta, \eta^{\prime}\right)
$$

for $\eta>\eta^{\prime}$ with initial condition $G_{n m}\left(\eta^{\prime}, \eta^{\prime}\right)=\delta_{n m}$ numerically using standard methods. Note that with $\boldsymbol{k}=k \boldsymbol{e}_{3}$ aligned with the three direction and $i k^{[0]}=-k$, the matrix $A_{n p}(\eta)$ is real and therefore the Green functions are real as well.

In principle, Green functions could be used to calculate all two-point functions for any $\eta$ until today, but generating the Green functions for late times and large multipoles 
numerically is very time-consuming. A much better performance can be achieved by combining this ansatz with the line-of-sight integration. Using the Green function method we compute $\Delta_{p}^{(2)}(\eta)$ for $l \leq 2$ and for early times, and then use Eq. (3.6) (with lower limit $\eta=0$ replaced by $\eta_{\text {ini }}$, since the visibility function is negligibly small at very early times) to obtain the late-time evolution of the higher multipole moments:

$$
\begin{aligned}
\Delta_{n}^{(2)}\left(\eta_{0}\right)= & \int_{\eta_{\mathrm{ini}}}^{\eta_{0}} d \eta|\dot{\kappa}(\eta)| e^{-\kappa(\eta)} j_{n m}\left(k\left(\eta_{0}-\eta\right)\right)\left[\varsigma_{m p} G_{p q}\left(\eta, \eta_{\mathrm{ini}}\right) \Delta_{q}^{(2)}\left(\eta_{\mathrm{ini}}\right)\right. \\
& \left.+\int_{\eta_{\mathrm{ini}}}^{\eta} d \eta^{\prime}\left(\delta_{m q} \delta\left(\eta-\eta^{\prime}\right)+\left|\dot{\kappa}\left(\eta^{\prime}\right)\right| \varsigma_{m p} G_{p q}\left(\eta, \eta^{\prime}\right)\right) S_{q}\left(\eta^{\prime}\right)\right] .
\end{aligned}
$$

Note that besides $\Delta_{q}^{(2)}\left(\eta_{\text {ini }}\right)$ only the source $S_{q}\left(\eta^{\prime}\right)$ depends on the stochastic initial conditions whereas the evolution is given by the non-stochastic functions $j_{n m}$ and $G_{p q}$. This separation is convenient for computing second-order correlation functions as described in the following subsections.

\subsection{Second-order power spectra}

The result Eq. (3.11) simplifies further if the initial second-order quantities $\Delta_{q}^{(2)}\left(\eta_{\text {ini }}\right)$ vanish. As we will discuss in section 4.2, this is the case for the computation of the nonscalar modes with $m= \pm 1, \pm 2$ and only these are needed in Eq. (2.15) to compute the B-mode angular power-spectrum. Consequently, we evaluate the two-point function assuming $\Delta_{q}^{(2)}\left(\eta_{\text {ini }}\right)=0$ and obtain

$$
\begin{aligned}
\left\langle\Delta_{n}^{(2)}(\boldsymbol{k},\right. & \left.\left.\eta_{0}\right) \Delta_{n^{\prime}}^{(2) *}\left(\boldsymbol{k}^{\prime}, \eta_{0}\right)\right\rangle \\
= & \int_{\eta_{\mathrm{ini}}}^{\eta_{0}} d \eta \int_{\eta_{\mathrm{ini}}}^{\eta_{0}} d \eta^{\prime}|\dot{\kappa}(\eta)|\left|\dot{\kappa}\left(\eta^{\prime}\right)\right| e^{-\kappa(\eta)-\kappa\left(\eta^{\prime}\right)} j_{n m}\left(k\left(\eta_{0}-\eta\right)\right) j_{n^{\prime} m^{\prime}}\left(k\left(\eta_{0}-\eta^{\prime}\right)\right) \\
& \times \int_{\eta_{\mathrm{ini}}}^{\eta} d \eta_{1} \int_{\eta_{\mathrm{ini}}}^{\eta^{\prime}} d \eta_{1}^{\prime}\left(\delta_{m q} \delta\left(\eta-\eta_{1}\right)+\left|\dot{\kappa}\left(\eta_{1}\right)\right| \varsigma_{m p} G_{p q}\left(\eta, \eta_{1}\right)\right) \\
& \times\left(\delta_{m^{\prime} q^{\prime}} \delta\left(\eta^{\prime}-\eta_{1}^{\prime}\right)+\left|\dot{\kappa}\left(\eta_{1}^{\prime}\right)\right| \varsigma_{m^{\prime} p^{\prime}} G_{p^{\prime} q^{\prime}}\left(\eta^{\prime}, \eta_{1}^{\prime}\right)\right)\left\langle S_{q}\left(\boldsymbol{k}, \eta_{1}\right) S_{q^{\prime}}^{*}\left(\boldsymbol{k}^{\prime}, \eta_{1}^{\prime}\right)\right\rangle,
\end{aligned}
$$

where we anticipated Eq. (3.16) below that sets $\boldsymbol{k}=\boldsymbol{k}^{\prime}$ in the integrand. We also imply that the helicity axis is the direction of $\boldsymbol{k}$ or, equivalently, that $\boldsymbol{k}$ is aligned with the threeaxis of the coordinate system. Comparison with Eq. (2.13) provides the power spectrum of $\Delta_{B}^{(2)}$ required to compute the B-mode angular power spectrum (2.15). The source term correlation function $\left\langle S_{q}\left(\boldsymbol{k}, \eta_{1}\right) S_{q^{\prime}}^{*}\left(\boldsymbol{k}^{\prime}, \eta_{1}^{\prime}\right)\right\rangle$ can be calculated from first-order quantities only. These are given by the primordial potential $\Phi(\boldsymbol{k}) \equiv A^{(1)}\left(\boldsymbol{k}, \eta_{\text {ini }}\right)$, multiplied by a transfer function $T$. Using $\boldsymbol{k}_{2}=\boldsymbol{k}-\boldsymbol{k}_{1}$ we can write

$$
\begin{aligned}
S_{n}(\boldsymbol{k}, \eta) & =\int \frac{d^{3} k_{1}}{(2 \pi)^{3}} K_{n}^{p q} \Delta_{p}^{(1)}\left(\boldsymbol{k}_{1}, \eta\right) \Delta_{q}^{(1)}\left(\boldsymbol{k}_{2}, \eta\right) \\
& =\int \frac{d^{3} k_{1}}{(2 \pi)^{3}} K_{n}^{p q} T_{p}^{(1)}\left(\boldsymbol{k}_{1}, \eta\right) T_{q}^{(1)}\left(\boldsymbol{k}_{2}, \eta\right) \Phi\left(\boldsymbol{k}_{1}\right) \Phi\left(\boldsymbol{k}_{2}\right)
\end{aligned}
$$


where the constants $K_{n}^{p q}$ are determined by the expressions for the second-order collision term, Eqs. (2.18) to (2.21). Thus, to compute the expectation value on the right-hand side of Eq. (3.12) we need to evaluate a four-point function of the primordial potential

$$
\begin{aligned}
\langle\Phi & \left.\left(\boldsymbol{k}_{1}\right) \Phi\left(\boldsymbol{k}_{2}\right) \Phi^{*}\left(\boldsymbol{k}_{1}^{\prime}\right) \Phi^{*}\left(\boldsymbol{k}_{2}^{\prime}\right)\right\rangle \\
= & \left\langle\Phi\left(\boldsymbol{k}_{1}\right) \Phi\left(\boldsymbol{k}_{2}\right)\right\rangle\left\langle\Phi^{*}\left(\boldsymbol{k}_{1}^{\prime}\right) \Phi^{*}\left(\boldsymbol{k}_{2}^{\prime}\right)\right\rangle+\left\langle\Phi\left(\boldsymbol{k}_{1}\right) \Phi^{*}\left(\boldsymbol{k}_{1}^{\prime}\right)\right\rangle\left\langle\Phi\left(\boldsymbol{k}_{2}\right) \Phi^{*}\left(\boldsymbol{k}_{2}^{\prime}\right)\right\rangle \\
& +\left\langle\Phi\left(\boldsymbol{k}_{1}\right) \Phi^{*}\left(\boldsymbol{k}_{2}^{\prime}\right)\right\rangle\left\langle\Phi\left(\boldsymbol{k}_{2}\right) \Phi^{*}\left(\boldsymbol{k}_{1}^{\prime}\right)\right\rangle \\
= & (2 \pi)^{6}\left(P_{\Phi}\left(k_{1}\right) P_{\Phi}\left(k_{1}^{\prime}\right) \delta^{(3)}(\boldsymbol{k}) \delta^{(3)}\left(\boldsymbol{k}^{\prime}\right)\right. \\
& \left.+P_{\Phi}\left(k_{1}\right) P_{\Phi}\left(k_{2}\right)\left[\delta^{(3)}\left(\boldsymbol{k}_{1}-\boldsymbol{k}_{1}^{\prime}\right) \delta^{(3)}\left(\boldsymbol{k}_{2}-\boldsymbol{k}_{2}^{\prime}\right)+\delta^{(3)}\left(\boldsymbol{k}_{1}-\boldsymbol{k}_{2}^{\prime}\right) \delta^{(3)}\left(\boldsymbol{k}_{2}-\boldsymbol{k}_{1}^{\prime}\right)\right]\right),
\end{aligned}
$$

where $P_{\Phi}$ is the primordial power spectrum, $\left\langle\Phi(\boldsymbol{k}) \Phi^{*}\left(\boldsymbol{k}^{\prime}\right)\right\rangle=(2 \pi)^{3} \delta^{(3)}\left(\boldsymbol{k}-\boldsymbol{k}^{\prime}\right) P_{\Phi}(k)$, and Gaussian statistics of the primordial potential $\Phi(\boldsymbol{k})$ was assumed. For $\boldsymbol{k} \neq 0$ or $\boldsymbol{k}^{\prime} \neq 0$ this results in

$$
\left\langle\Phi\left(\boldsymbol{k}_{1}\right) \Phi\left(\boldsymbol{k}_{2}\right) \Phi^{*}\left(\boldsymbol{k}_{1}^{\prime}\right) \Phi^{*}\left(\boldsymbol{k}_{2}^{\prime}\right)\right\rangle=(2 \pi)^{6} P_{\Phi}\left(k_{1}\right) P_{\Phi}\left(k_{2}\right) \delta^{(3)}\left(\boldsymbol{k}-\boldsymbol{k}^{\prime}\right)\left[\delta^{(3)}\left(\boldsymbol{k}_{1}-\boldsymbol{k}_{1}^{\prime}\right)+\delta^{(3)}\left(\boldsymbol{k}_{1}-\boldsymbol{k}_{2}^{\prime}\right)\right],
$$

and the complete expectation value on the right-hand side of Eq. (3.12) reads

$$
\begin{aligned}
\left\langle S_{n}(\boldsymbol{k}, \eta) S_{n^{\prime}}^{*}\left(\boldsymbol{k}^{\prime}, \eta^{\prime}\right)\right\rangle= & (2 \pi)^{3} \delta^{(3)}\left(\boldsymbol{k}-\boldsymbol{k}^{\prime}\right) \int \frac{d^{3} k_{1}}{(2 \pi)^{3}} P_{\Phi}\left(k_{1}\right) P_{\Phi}\left(k_{2}\right) K_{n}^{p q} K_{n^{\prime}}^{p^{\prime} q^{\prime} *} \\
\times & {\left[T_{p}^{(1)}\left(\boldsymbol{k}_{1}, \eta\right) T_{q}^{(1)}\left(\boldsymbol{k}_{2}, \eta\right) T_{p^{\prime}}^{(1) *}\left(\boldsymbol{k}_{1}, \eta^{\prime}\right) T_{q^{\prime}}^{(1) *}\left(\boldsymbol{k}_{2}, \eta^{\prime}\right)\right.} \\
& \left.+T_{p}^{(1)}\left(\boldsymbol{k}_{1}, \eta\right) T_{q}^{(1)}\left(\boldsymbol{k}_{2}, \eta\right) T_{p^{\prime}}^{(1) *}\left(\boldsymbol{k}_{2}, \eta^{\prime}\right) T_{q^{\prime}}^{(1) *}\left(\boldsymbol{k}_{1}, \eta^{\prime}\right)\right] .
\end{aligned}
$$

For a given primordial spectrum, we can now compute the second-order power spectrum using Eq. (3.12) and Eq. (3.16).

\subsection{Non-Gaussianity}

Analogously, the method described above can be applied to study non-Gaussianity. At first order, the bispectrum (three-point function) is always proportional to the primordial bispectrum and therefore zero if the primordial perturbations are Gaussian. This is different, if non-linear effects are taken into account. The leading contributions to the bispectrum are terms combining one second-order perturbation with two first-order perturbations,

$$
\left\langle\Delta_{n} \Delta_{m} \Delta_{p}\right\rangle=\left\langle\Delta_{n}^{(2)} \Delta_{m}^{(1)} \Delta_{p}^{(1)}\right\rangle+\mathrm{sym}
$$

We replace the first-order quantities by their transfer functions and the primordial potential and calculate the second-order quantity using the combination of line-of-sight integration and Green functions, Eq. (3.11). Allowing for non-vanishing initial values $\Delta_{n}^{(2)}\left(\boldsymbol{k}, \eta_{\text {ini }}\right)$ we 
find, for $\boldsymbol{k}$ aligned with the three-axis of the coordinate system,

$$
\begin{aligned}
& \left\langle\Delta_{n}^{(2)}\left(\boldsymbol{k}, \eta_{0}\right) \Delta_{q}^{(1)}\left(\boldsymbol{k}^{\prime}, \eta_{0}\right) \Delta_{r}^{(1)}\left(\boldsymbol{k}^{\prime \prime}, \eta_{0}\right)\right\rangle=T_{q}^{(1)}\left(\boldsymbol{k}^{\prime}, \eta_{0}\right) T_{r}^{(1)}\left(\boldsymbol{k}^{\prime \prime}, \eta_{0}\right) \\
& \quad \times \int_{\eta_{\mathrm{ini}}}^{\eta_{0}} d \eta|\dot{\kappa}| e^{-\kappa} j_{n m}\left(k\left(\eta_{0}-\eta\right)\right)\left[\varsigma_{m p} G_{p q}\left(\eta, \eta_{\mathrm{ini}}\right)\left\langle\Delta_{q}^{(2)}\left(\boldsymbol{k}, \eta_{\mathrm{ini}}\right) \Phi\left(\boldsymbol{k}^{\prime}\right) \Phi\left(\boldsymbol{k}^{\prime \prime}\right)\right\rangle\right. \\
& \left.\quad+\int_{\eta_{\mathrm{ini}}}^{\eta} d \eta^{\prime}\left(\delta_{m q} \delta\left(\eta-\eta^{\prime}\right)+\left|\dot{\kappa}\left(\eta^{\prime}\right)\right| \varsigma_{m p} G_{p q}\left(\eta, \eta^{\prime}\right)\right)\left\langle S_{q}\left(\boldsymbol{k}, \eta^{\prime}\right) \Phi\left(\boldsymbol{k}^{\prime}\right) \Phi\left(\boldsymbol{k}^{\prime \prime}\right)\right\rangle\right] .
\end{aligned}
$$

Using Eq. (3.13) and contracting the resulting four-point function similar to Eq. (3.14) we can write the second expectation value as

$$
\begin{aligned}
\left\langle S_{n}\left(\boldsymbol{k}, \eta^{\prime}\right) \phi\left(\boldsymbol{k}^{\prime}\right) \phi\left(\boldsymbol{k}^{\prime \prime}\right)\right\rangle=(2 \pi)^{3} \delta^{(3)}\left(\boldsymbol{k}+\boldsymbol{k}^{\prime}+\boldsymbol{k}^{\prime \prime}\right) P_{\Phi}\left(k^{\prime}\right) P_{\Phi}\left(k^{\prime \prime}\right) K_{n}^{p q} \\
\times\left[T_{p}^{(1)}\left(-\boldsymbol{k}^{\prime}, \eta^{\prime}\right) T_{q}^{(1)}\left(-\boldsymbol{k}^{\prime \prime}, \eta^{\prime}\right)+T_{p}^{(1)}\left(-\boldsymbol{k}^{\prime \prime}, \eta^{\prime}\right) T_{q}^{(1)}\left(-\boldsymbol{k}^{\prime}, \eta^{\prime}\right)\right] .
\end{aligned}
$$

For an initial value $\Delta_{q}^{(2)}\left(\boldsymbol{k}, \eta_{\text {ini }}\right)$ which is quadratic in the primordial potential $\Phi$, also the first expectation value can be contracted and written in terms of the primordial powerspectrum. A detailed study of non-Gaussianity from second-order effects employing these equations will be presented in a follow-up article.

\section{Numerical evaluation}

In this section we outline the steps required to compute the two-point function (3.12) and the $C_{l}^{B B}$ angular power spectrum (2.15) numerically. First we need to compute the first-order transfer functions, which is discussed in Section 4.1, and the Green functions, as discussed in Section 4.3. In Section 4.5 we obtain the source terms and combine both results to perform the line-of-sight integral together with the wave-vector convolutions.

For all intermediate results presented in this section we use a $\Lambda \mathrm{CDM}$ model with massless neutrinos and the following parameters: $T_{\mathrm{CBR}}=2.726 \mathrm{~K}, H_{0}=70 \mathrm{~km} /(\mathrm{s} \mathrm{Mpc})$, and $\Omega_{\mathrm{CDM}}=0.245, \Omega_{\text {baryon }}=0.045, \Omega_{\Lambda}=0.71$. We assume a scale-invariant primordial power-spectrum $P_{\Phi}(k)=2 \pi^{2}(9 / 25) \Delta_{\mathcal{R}}^{2} / k^{3}$ with amplitude $\Delta_{\mathcal{R}}^{2}=2.41 \times 10^{-9}$. To compute the recombination history we employ RECFAST [16] with a helium mass-fraction $Y_{\mathrm{p}}=0.24$. Our calculation does not include late effects such as the late integrated Sachs-Wolfe effect (ISW) and reionization. Scattering effects and the early ISW will be considered up to $\eta=$ $500 \mathrm{Mpc} / c$ and neglected for later times where we use the free-streaming approximation. Recombination occurs around $\eta_{\text {rec }}=286.7 \mathrm{Mpc} / c$, defined as the median of the visibility function.

\subsection{First-order solutions}

The first-order transfer functions can be computed by solving the first-order Boltzmann equations with standard methods for solving ordinary differential equations (e.g. as implemented in the GNU Scientific Library [17]). Alternatively one can resort to programs like Cmbfast [14], Camb [18] or CMbeasy [19]. The following results are based on our own code that has been compared to CAMB. 
A crucial point is that we need only the low multipoles at early times where the numerical calculation is straightforward. The restriction to early times is due to the factor $|\dot{\kappa}|$ in all collision term sources which is negligible after recombination. The reason why only low multipoles are needed at early times is that higher moments are only slowly generated by free streaming. Thus, one can cut the Boltzmann hierarchy at some $l_{\text {cut }}$ and neglect all higher multipole moments. Alternatively, to save CPU time, one can apply the cut at a much lower multipole where the corresponding moment cannot be neglected, but is replaced by a closing relation. This option will be discussed in detail in Section 4.4 .

Neutrinos start free streaming much earlier than photons because they are not tightly coupled to baryons by Thomson scattering and thus start generating higher multipoles at early times. This has to be taken into account by choosing a cut at larger $l$ for the neutrino hierarchy than for the photon hierarchy. Typically we take more than 50 neutrino multipoles into account which yields accurate results until matter domination.

\subsection{Initial conditions}

We assume that the primordial first-order perturbations are adiabatic and begin the evolution of the transfer functions at $a_{\mathrm{ini}}=10^{-6}$ (corresponding to $\eta_{\mathrm{ini}}=0.464 \mathrm{Mpc} / \mathrm{c}$ ) deep in the radiation era with standard adiabatic initial conditions for the scalar perturbations:

$$
\begin{aligned}
& T_{A}^{(1)}(k) \equiv T_{\Phi}^{(1)}(k)=1, \quad T_{D}^{(1)}(k)=-\left(1+\frac{2}{5} \frac{\bar{\rho}_{\nu}}{\bar{\rho}_{\gamma}+\bar{\rho}_{\nu}}\right), \\
& T_{\delta_{b}}^{(1)}(k)=T_{\delta_{c}}^{(1)}(k)=\frac{3}{4} T_{\Delta_{I, 00}}^{(1)}(k)=\frac{3}{4} T_{\Delta_{\nu, 00}}^{(1)}(k)=-\frac{3}{2}, \\
& T_{v_{e,[0]}}^{(1)}(k)=T_{v_{c,[0]}}^{(1)}(k)=\frac{1}{4} T_{\Delta_{I, 10}}^{(1)}(k)=\frac{1}{4} T_{\Delta_{\nu, 10}}^{(1)}(k)=\frac{k}{2 H_{C}} .
\end{aligned}
$$

For the neutrinos we further include an initial quadrupole $T_{\Delta_{\nu, 20}}^{(1)}(k)=2 k^{2} /\left(3 H_{C}^{2}\right)$, which however is very small at $\eta_{\text {ini }}$, when for all $k$ of interest $k / H_{C} \ll 1$.

Setting the initial conditions for the second-order perturbation variables is simplified by the fact that by Eq. (2.15) we only need to compute the vector and tensor perturbations $m= \pm 1, \pm 2$ when working in the frame where the mode-vector $\boldsymbol{k}$ is aligned with the helicity axis (usually the three-direction). While we may adopt the convention that at some initial time $A^{(2)}$ vanishes, since any non-zero value can be absorbed into a small change of $A^{(1)}$, this cannot generally be done for all perturbation variables. For instance, deep in the radiation era, when $k / H_{C} \ll 1$, the total energy density perturbation is given by

$$
\frac{\delta \rho^{(2)}}{\bar{\rho}}=-2 A^{(2)}+4 H_{C}\left[A^{(1)}\right]^{2} .
$$

However, the equations we need to solve for $m= \pm 1, \pm 2$ do not contain the second-order energy density perturbations, that is the monopoles of the multipole decomposition, and hence we do not have to determine their initial conditions.

We do need the $m= \pm 1$ components of the second-order electron velocity $v_{e,[m]}^{(2)}$ and the radiation intensity dipole. Their initial values are related to the metric vector perturbation

$B_{[m]}^{(2)}$. Since, as discussed above, we do not consider the second-order metric perturbations 
in this paper, the initial value of the second-order velocities and dipoles is consistently set to zero. In the tightly coupled radiation era there exists a non-vanishing radiation quadrupole $\Delta_{I, 2 m}^{(2)}$, which acquires a non-zero initial condition given in terms of the square of the first-order electron velocity. Being of second order and suppressed by $\left(k / H_{C}\right)^{2}$, this can be safely neglected. We show in Section 4.6 that collisions quickly drive the quadrupole to its tight-coupling value when it is zero initially. Finally, we note that the second-order neutrino perturbations do not appear in our equations, so we do not have to set their initial values.

To sum up, we may solve the second-order equations with all initial values of secondorder variables set to zero.

\subsection{Green functions}

The differential equations (3.10) for the Green functions are far less complicated than the original second-order equations. In particular, the equations are no longer stochastic. Their structure is identical to the one of the first-order Boltzmann equations.

The relevant Green functions $G_{n m}\left(\eta, \eta^{\prime}\right)$ can be classified by several criteria. All Green functions except those for monopole, dipole, quadrupole and electron velocity are strongly tight-coupling suppressed. ${ }^{1}$ This leads to a suppression of these Green functions if $\eta^{\prime}$ is located before recombination (except for $\eta$ close to $\eta^{\prime}$ ), and allows us to restrict the $\eta^{\prime}$ integration to a period directly around recombination, as after recombination the source terms vanish with $|\dot{\kappa}|$ and before recombination the Green function is small, even after multiplication with the large scattering rate. The effect resembles that of the visibility function $|\dot{\kappa}| e^{-\kappa}$ in the line-of-sight integration which is peaked around recombination.

Green functions which are not suppressed during tight-coupling may lead to numerical difficulties because they in turn do not suppress the source $\sigma_{n}=|\dot{\kappa}| S_{n}$ in Eq. (3.8). At early times, the scattering rate $|\dot{\kappa}|$ is huge and, multiplied with the numerical error of $S_{n}$, yields a large absolute error of $\sigma_{n}$ and the corresponding second-order quantities. As a consequence, linear combinations of these second-order quantities can obtain a large relative error, if there exist cancellations. For the electron velocity and radiation dipole Green functions such problems can be avoided by exploiting the close relation of dipole and electron-velocity sources. The electron-velocity sources can be split into two parts, one which is - apart from a prefactor - identical to the photon-intensity dipole source and

a remaining part $\tilde{S}_{v_{e}, 1 m}$ which cancels the factor of $|\dot{\kappa}|$ by the tight-coupling suppression $1 /|\dot{\kappa}|$ of the combination of first-order perturbations multiplying it. Writing

$$
S_{v_{e}, 1 m}=-\frac{1}{4 R} S_{I, 1 m}+\tilde{S}_{v_{e}, 1 m}
$$

where

$$
\tilde{S}_{v_{e}, 1 m}=\frac{1}{4 R} \delta_{b}^{(1)}\left(\boldsymbol{k}_{1}\right)\left(4 v_{e,[m]}^{(1)}\left(\boldsymbol{k}_{2}\right)-\Delta_{I, 1 m}^{(1)}\left(\boldsymbol{k}_{2}\right)\right),
$$

\footnotetext{
${ }^{1}$ This does not apply to the corresponding calculation of second-order neutrino perturbations.
} 


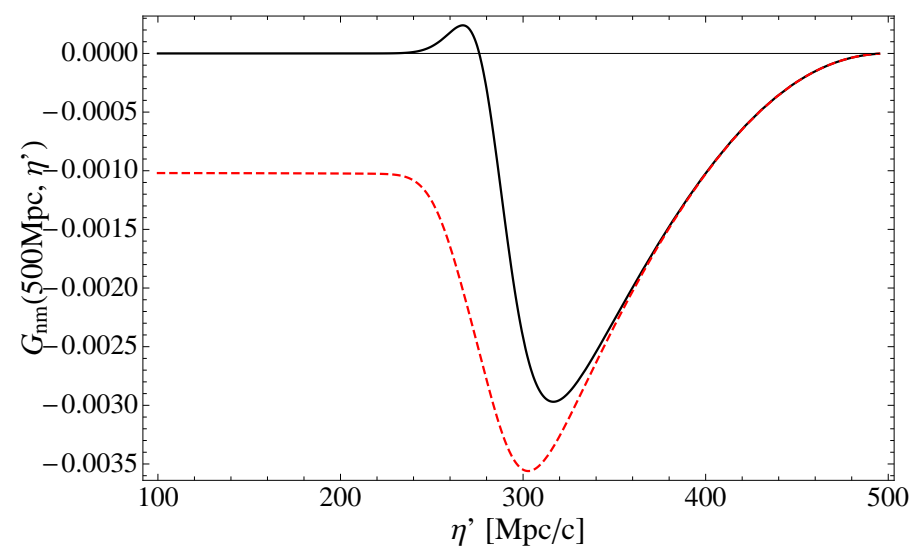

Figure 1: In dashed (red) the Green function $G_{(E, 21),(I, 11)}\left(500 \mathrm{Mpc} / c, \eta^{\prime}\right)$ for $\boldsymbol{k}=\boldsymbol{k} \boldsymbol{e}_{3}$, $k=0.02 \mathrm{Mpc}^{-1}$. Since the dipole is not tight-coupling suppressed, the Green function does not vanish before recombination. After recombination the Green function vanishes because $\Delta_{E, 21}$ and $\Delta_{I, 11}$ are only coupled in the scattering term. The solid (black) line is the combined dipole and electron-velocity Green function $G_{(E, 21),(I, 11)}\left(500 \mathrm{Mpc} / c, \eta^{\prime}\right)-\frac{1}{4 R} G_{(E, 21),\left(v_{e}, 11\right)}\left(500 \mathrm{Mpc} / c, \eta^{\prime}\right)$ which vanishes at early times.

the first part on the right-hand side of Eq. (4.3) can be combined with the photon source in Eq. (3.8) to obtain

$$
\begin{aligned}
\Delta_{n}^{(2)}(\eta)= & \int_{\eta_{\text {ini }}}^{\eta} d \eta^{\prime}\left|\dot{\kappa}\left(\eta^{\prime}\right)\right|\left(G_{n,(I, 1 m)}\left(\eta, \eta^{\prime}\right) S_{I, 1 m}\left(\eta^{\prime}\right)+G_{n,\left(v_{e}, 1 m\right)}\left(\eta, \eta^{\prime}\right) S_{v_{e}, 1 m}\left(\eta^{\prime}\right)\right) \\
& +\sum_{\text {all other } p} \int_{\eta_{\text {ini }}}^{\eta} d \eta^{\prime}\left|\dot{\kappa}\left(\eta^{\prime}\right)\right| G_{n p}\left(\eta, \eta^{\prime}\right) S_{p}\left(\eta^{\prime}\right) \\
= & \int_{\eta_{\text {ini }}}^{\eta} d \eta^{\prime}\left|\dot{\kappa}\left(\eta^{\prime}\right)\right|\left(G_{n,(I, 1 m)}\left(\eta, \eta^{\prime}\right)-\frac{1}{4 R} G_{n,\left(v_{e}, 1 m\right)}\left(\eta, \eta^{\prime}\right)\right) S_{I, 1 m}\left(\eta^{\prime}\right) \\
& +\int_{\eta_{\text {ini }}}^{\eta} d \eta^{\prime}\left|\dot{\kappa}\left(\eta^{\prime}\right)\right| G_{n,\left(v_{e}, 1 m\right)}\left(\eta, \eta^{\prime}\right) \tilde{S}_{v_{e}, 1 m}\left(\eta^{\prime}\right) \\
& +\sum_{\text {all other } p} \int_{\eta_{\text {ini }}}^{\eta} d \eta^{\prime}\left|\dot{\kappa}\left(\eta^{\prime}\right)\right| G_{n p}\left(\eta, \eta^{\prime}\right) S_{p}\left(\eta^{\prime}\right)
\end{aligned}
$$

Thus, only the combination $G_{n,(I, 1 m)}-\frac{1}{4 R} G_{n,\left(v_{e}, 1 m\right)}$ is multiplied with the source $S_{I, 1 m}$ which is large at early times, but this combination of Green functions does vanish in the tight-coupling regime. In Fig. 1 the combination is plotted illustrating the suppression during tight-coupling (solid line).

The remaining Green functions which do not vanish in tight-coupling after multiplication with $|\dot{\kappa}|$ include: the Green function acting on the photon monopole, the one acting on the remaining part of the electron-velocity source $\tilde{S}_{v_{e}, 1 m}$ and the Green functions acting on the quadrupoles. The first does not enter our computation since the photon monopole does not couple to polarization, the second is suppressed by $\tilde{S}_{v_{e}, 1 m}$ which itself is tight-coupling suppressed as explained above. Finally, the Green functions acting on the quadrupoles are 


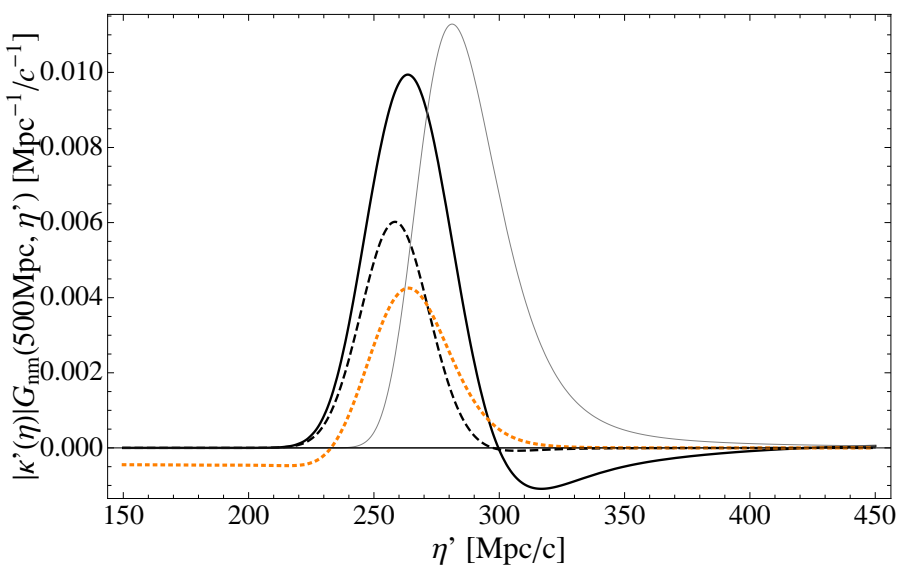

Figure 2: Green functions $G_{(E, 22),(E, 22)}\left(500 \mathrm{Mpc} / c, \eta^{\prime}\right), 10 \times G_{(I, 22),(E, 22)}\left(500 \mathrm{Mpc} / c, \eta^{\prime}\right)$ and $G_{(I, 21),(E, 21)}\left(500 \mathrm{Mpc} / c, \eta^{\prime}\right)$ (solid/black, dashed/black and dotted/orange, respectively) for $\boldsymbol{k}=$ $k \boldsymbol{e}_{3}, k=0.03 \mathrm{Mpc}^{-1}$, multiplied with $\left|\dot{\kappa}\left(\eta^{\prime}\right)\right|$. The multipole moments $\Delta_{E, 2 m}$ and $\Delta_{I, 2 m}$ are only coupled in the scattering term. As expected, the Green function connecting these moments vanishes faster than the Green function connecting moments coupled in the free-streaming term. The Green function acting on quadrupoles for $|m|=1$ is only suppressed by $|\dot{\kappa}|$ and thus the plotted combination is constant at early times. The visibility function divided by two is shown in grey for comparison.

only suppressed by one power of $|\dot{\kappa}|$ for $|m|=1$, and do not vanish when multiplied by $|\dot{\kappa}|{ }^{2}$ Hence, while for $|m|=2$ the $\eta^{\prime}$ integration in Eq. (3.8) can be restricted to times around recombination as there are no contributions at early times, the time integration cannot be cut for $|m|=1$. This difference between vector and tensor perturbations is related to the persistence of the dipoles during tight-coupling. While during tight-coupling no polarization is generated, the short-lived excitation of a quadrupole will modify the dipoles. This dipole is then converted into a quadrupole during recombination which couples to polarization. This indirect coupling of early vector sources can induce polarization while early tensor sources have no influence, as there is no unsuppressed moment.

After recombination, the characteristics of a Green function depend on the coupling between the Green function's two multipoles. If both moments are only coupled in the scattering term, the Green function quickly vanishes after recombination. If they are coupled via free streaming, it oscillates, since free streaming converts neighboring moments into each other. In combination with the factor $\left|\dot{\kappa}\left(\eta^{\prime}\right)\right|$ in Eq. (3.11) both types of Green functions decay after recombination. However, the former decays with an additional factor of $|\dot{\kappa}|$, as depicted in Fig. 2 .

\subsection{Closing relations}

Like the first-order Boltzmann hierarchy, the equations for the Green functions need to be truncated at some multipole moment $l_{\text {cut }}$. The straightforward approach is to set higher moments with $l>l_{\text {cut }}$ to zero. This is a good approximation at early times because all

\footnotetext{
${ }^{2}$ The Green functions for $|m|=2$ are exponentially suppressed as there is no coupling to the unsuppressed dipole.
} 
higher moments are initially zero. But once the moment $l_{\text {cut }}$ is excited by free streaming, the solutions for the Green functions and first-order quantities, respectively, become inaccurate, since in the full hierarchy a non-zero moment at $l_{\text {cut }}$ excites the moment $l_{\text {cut }}+1$ which then feeds back into the lower moments. However, the error will first become noticeable only in the moments close to $l_{\text {cut }}$. It then takes an equal amount of time to carry the error back to the lowest multipoles as it has taken to excite $l_{\text {cut }}$ in the first place. Hence, one can still trust the results for the lowest multipoles some time after the highest multipole has been excited.

For accurate results one has to cut at some sufficiently large $l_{\text {cut }}$ such that the lowest moments (which we are interested in) are not influenced by the truncation. To compute the photon multipole $l$ at time $\eta$ and wave number $k$, the rule of thumb is to cut at $l_{\text {cut }} \approx l+k\left(\eta-\eta_{\text {rec }}\right) / 2$. Thus, if $k$ or $\eta$ is large, many multipoles have to be taken into account. This can be avoided by using appropriate closing relations at a comparably small $l$ [14].

Approximating the visibility function by a delta function at $\eta_{\text {rec }}$ allows us to derive an analytic relation between the highest multipoles we consider. In this approximation the scattering rate is infinitely large before recombination so that all multipoles except for the monopole and dipole are zero due to tight-coupling suppression. After recombination, the scattering rate and therefore the sources are zero and we can employ the free-streaming solution Eq. (B.10) to obtain

$$
\begin{aligned}
\Delta_{X, l m}(\eta)= & \sum_{l_{2}=0,1} \sum_{X^{\prime}, l_{1}} i^{l-l_{1}-l_{2}} \frac{(2 l+1)\left(2 l_{1}+1\right)}{2 l_{2}+1} j_{l_{1}}\left(k\left(\eta-\eta_{\mathrm{rec}}\right)\right) \\
& \times\left(\begin{array}{ccc}
l & l_{1} & l_{2} \\
m & 0 & m
\end{array}\right)\left(\begin{array}{ccc}
l & l_{1} & l_{2} \\
F_{X^{\prime}} & 0 & F_{X^{\prime}}
\end{array}\right) H_{X X^{\prime}}^{*}\left(l-l_{1}-l_{2}\right) \Delta_{X^{\prime}, l_{2} m}\left(\eta_{\mathrm{rec}}\right) .
\end{aligned}
$$

Starting from this equation we perform the following steps to derive the closing relations, exemplified here for the intensity multipoles $\Delta_{I, l 0}$ and $l_{\text {cut }}=9 .^{3}$

- We write down Eq. (4.6) explicitly for the two highest multipole moments below $l_{\text {cut }}$, $\Delta_{X,\left(l_{\mathrm{cut}}-1\right) m}$ and $\Delta_{X,\left(l_{\mathrm{cut}}-2\right) m}$ :

$$
\begin{aligned}
& \Delta_{I, 80}(\eta)=\left(8 j_{7}-9 j_{9}\right) \Delta_{I, 10}\left(\eta_{\mathrm{rec}}\right)+17 j_{8} \Delta_{I, 00}\left(\eta_{\mathrm{rec}}\right) \\
& \Delta_{I, 70}(\eta)=\left(7 j_{7}-8 j_{9}\right) \Delta_{I, 10}\left(\eta_{\mathrm{rec}}\right)+15 j_{8} \Delta_{I, 00}\left(\eta_{\mathrm{rec}}\right)
\end{aligned}
$$

For brevity we leave out the argument of the Bessel functions which is always $k(\eta-$ $\eta_{\text {rec }}$ ). For $m \neq 0$, the monopole (and even the dipole for $m= \pm 2$ ) does not exist, and one uses instead the two lowest non-vanishing multipoles. For polarization, one uses the E- and B-mode quadrupole.

- We solve both equations to write the monopole and dipole at recombination in terms of the multipoles $\Delta_{X,\left(l_{\mathrm{cut}}-1\right) m}$ and $\Delta_{X,\left(l_{\mathrm{cut}}-2\right) m}$. In case of polarization $(X=E, B)$,

\footnotetext{
${ }^{3}$ The $m=0$ calculation is not required to compute B-mode polarization, but the expressions are less complicated and the method can be extended straightforwardly to other values of $m$.
} 
we have to relate $\Delta_{E,\left(l_{\text {cut }}-1\right) m}$ and $\Delta_{B,\left(l_{\text {cut }}-1\right) m}$ to $\Delta_{E, 2 m}$ and $\Delta_{B, 2 m}$ instead, due to the mixing of $E$ modes and $B$ modes during free streaming.

- In Eq. (4.6) for $\Delta_{X, l_{\mathrm{cut}} m}$ we can now replace the monopole and dipole on the righthand side with the result from the previous step to obtain the closing relation. In our example we find

$$
\begin{aligned}
\Delta_{I, 90}(\eta)= & \frac{19 j_{9}\left(-7 j_{6}+8 j_{8}\right)+15 j_{7}\left(9 j_{8}-10 j_{10}\right)}{17 j_{8}\left(-7 j_{6}+8 j_{8}\right)+15 j_{7}\left(8 j_{7}-9 j_{9}\right)} \Delta_{I, 80}(\eta) \\
& +\frac{19 j_{9}\left(8 j_{7}-9 j_{9}\right)+17 j_{8}\left(-9 j_{8}+10 j_{10}\right)}{17 j_{8}\left(-7 j_{6}+8 j_{8}\right)+15 j_{7}\left(8 j_{7}-9 j_{9}\right)} \Delta_{I, 70}(\eta) .
\end{aligned}
$$

- Finally, we use this result to replace $\Delta_{X, l_{\text {cut }} m}$ in the Boltzmann hierarchy and obtain a closed system of differential equations for all moments up to $\Delta_{X,\left(l_{\mathrm{cut}}-1\right) m}$ which is independent of higher moments.

The quality of this approximation depends on the width of the visibility function. While the photon evolution is dominated by scattering effects, neutrino modes are exclusively sourced by metric terms. Consequently, the closing relations described here cannot be applied to neutrinos, since they rely on the sharply peaked visibility function. However, we only have to consider neutrino perturbations at first order, so that all Green functions can be computed using the closing relations.

In our calculation of the Green functions we cut the hierarchy at $l_{\text {cut }}=9$, obtaining the closing relation

$$
\Delta_{I, 9 m}(\eta)=h_{I, 8 m}\left(k\left(\eta-\eta_{\mathrm{rec}}\right)\right) \Delta_{I, 8 m}(\eta)+h_{I, 7 m}\left(k\left(\eta-\eta_{\mathrm{rec}}\right)\right) \Delta_{I, 7 m}(\eta) .
$$

In general the functions $h_{X, l m}$ are combinations of spherical Bessel functions as in the $m=0$ example, Eq. (4.8). For $m \neq 0$ the functions become more complicated, but as long as the argument $k\left(\eta-\eta_{\mathrm{rec}}\right)$ is small, they can be approximated by polynomials. This is not possible for larger arguments where the functions oscillate, see Fig. 3. Only for very large arguments the oscillations can be neglected due to damping and a simple approximation can be used again.

Figure 4 shows that the closing relations can be used to significantly reduce the error compared to a simple truncation — at almost no additional CPU time.

\subsection{Source terms and integration}

To compute the angular power spectrum $C_{l}$ at second order, we have to perform eight integrations: two time integrals from the line-of-sight solution, another two time integrals from the Green function ansatz, three integrations from the convolution over $\boldsymbol{k}_{1}$ and finally the $k$ integral in Eq. (2.15). The integral over the angular coordinate $\phi_{1}$ of the wave vector $\boldsymbol{k}_{1}$ can be performed analytically. The remaining seven integrals are computed in one single Monte Carlo integration. The wave-vector integrations need to be cut at some $k_{\max }$. The larger the choice of $k_{\max }$, the more multipoles have to be considered when computing firstorder results and Green functions, increasing the demand for CPU time. Fortunately it 


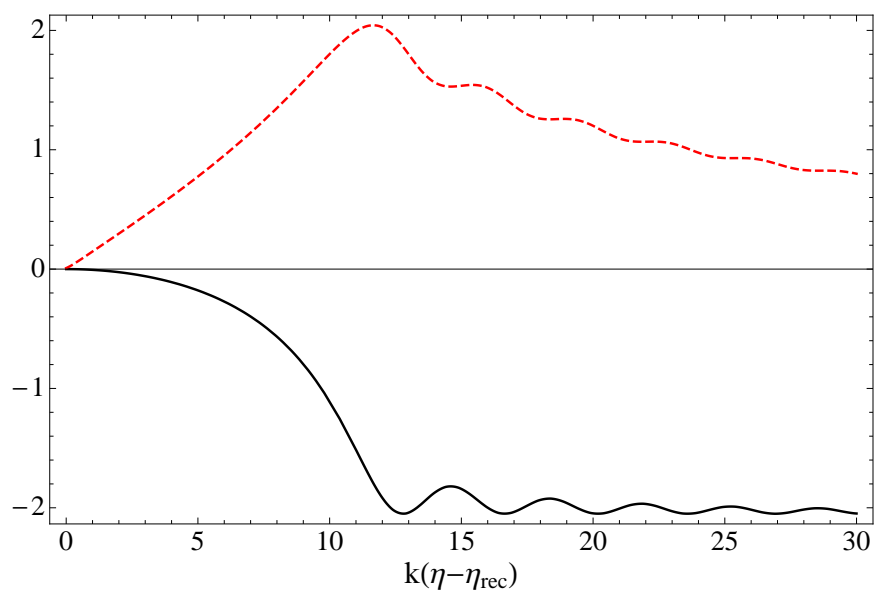

Figure 3: The functions $h_{I, 72}$ (solid/black) and $h_{I, 82}$ (dashed/red) which appear in the closing relation (4.9).

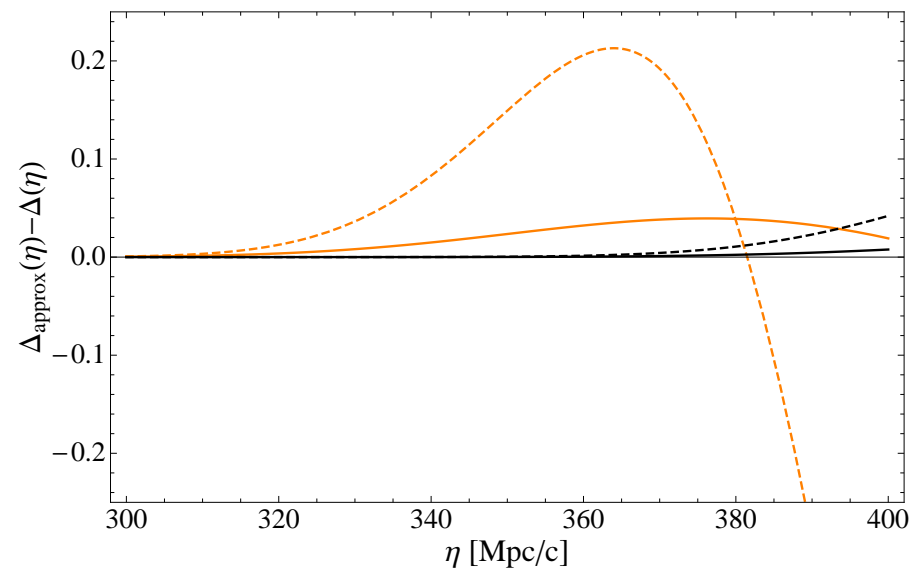

Figure 4: Comparison of the absolute error obtained by using a simple truncation (dashed) and the closing relations (solid) for $\Delta_{I 80}^{(1)}$ (black) and $\Delta_{I 20}^{(1)}$ (orange); $k=0.1 \mathrm{Mpc}^{-1}, l_{\text {cut }}=9$. The improvement achieved by using closing relations is even better for smaller wave numbers.

turns out that the integrand decays quickly for $k_{1}$ or $k_{2}$ larger than $k$. The $k_{1}$ dependence of the integrand is illustrated in Fig. 5, depicting the suppression for large values of $k_{1}$. This effect is due to the two primordial power spectra in the expectation value of the source terms, Eq. (3.16). Since $k_{2}=\left|\boldsymbol{k}-\boldsymbol{k}_{1}\right|$, for large $k_{1}$ the power spectra suppress the integrand with $\mathcal{O}\left(k_{1}^{-6}\right)$. We find that choosing $k_{\max }=0.3 \mathrm{Mpc}^{-1}$ is more than adequate, see Section 4.7.

Since we calculate only collisional sources which are multiplied by the visibility function, many time integrals could be restricted to $200 \mathrm{Mpc} / c \lesssim \eta \lesssim 500 \mathrm{Mpc} / c$. This also applies to the Green function integrals, where the integrand is tight-coupling suppressed as discussed in Section 4.3. However, in the numerical results presented below, we use $\eta_{\text {ini }}$ as lower bound for the time integrals. The adaptive Monte Carlo algorithm (VEgAs) [20] automatically samples fewer points between $\eta_{\text {ini }}$ and $200 \mathrm{Mpc} / c$ than for later times. The upper bound of the time integrations will be denoted by $\eta_{\mathrm{fs}}$. To verify that $\eta_{\mathrm{fs}}=500 \mathrm{Mpc} / \mathrm{c}$ 
is indeed a reasonable choice, we study the convergence of the result with increasing $\eta_{\mathrm{fs}}$, see Section 4.7.

Only source terms $S_{X, l m}$ with small multipole moment $l$ have to be taken into account, since higher first-order moments are only generated by free streaming which takes time. For typical values of $k$, the octupole reaches its first maximum only after $\eta_{\text {rec }}$, so that its contribution to the integrand which is peaked around recombination is small. Higher moments are generated even later. If Eq. (3.11) is used to compute the polarization modes, there is an additional suppression of the intensity sources $S_{I, l m}$ : since the function $j_{n m}$ mixes only the two polarization modes, the intensity source contributes to polarization solely via the Green functions in the second line of Eq. (3.11). But as stated in Section 4.3, a Green function $G_{m n}\left(\eta, \eta^{\prime}\right)$ for multipoles coupled only by the scattering term vanishes if the scattering rate $|\dot{\kappa}|$ goes to zero. Accordingly, due to the additional $|\dot{\kappa}|$ factor in front of the Green function, for small scattering rates the contribution of $S_{I, l m}$ sources is proportional to $|\dot{\kappa}|^{2}$ and thus suppressed much earlier than other contributions proportional to $|\dot{\kappa}|$. Hence, sources $S_{I, l m}$ with $l>2$ are not only tight-coupling suppressed at early times but in addition do not contribute at late times.

In the following we denote by $n_{l}$ the highest source-term multipole moment included. All sources $S_{X, l m}$ with $l>n_{l}$ are neglected. By the reasoning above we conclude that $n_{l}=3$ should already provide reasonable results, since $S_{X, 3 m}$ is the highest source that contains the first-order quadrupole. This will be verified in detail in Section 4.7. Note that the source $S_{X, n_{l} m}$ contains first-order solutions with $l=n_{l}+1$, i.e., for $n_{l}=3$ we need the

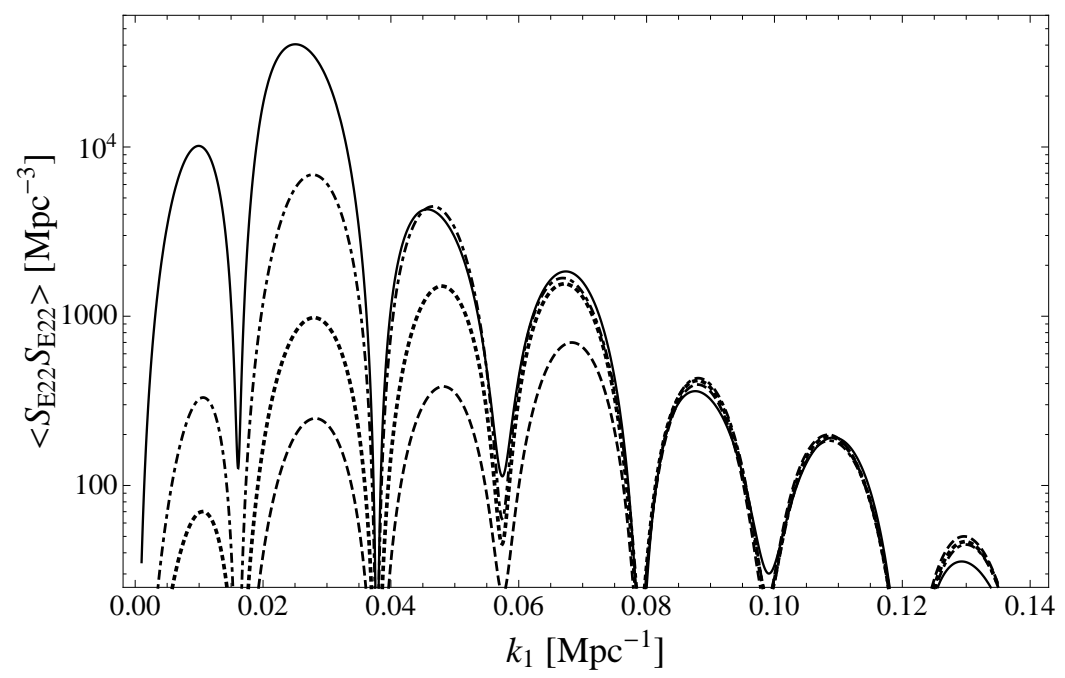

Figure 5: The $k_{1}$ integrand of $\left\langle S_{E, 22}(\boldsymbol{k}, \eta) S_{E, 22}^{*}\left(\boldsymbol{k}^{\prime}, \eta^{\prime}\right)\right\rangle$ (cf. Eq. (3.16)) integrated over $\boldsymbol{k}^{\prime}$. In the convolution integral over $\boldsymbol{k}_{1}$ we have only performed the integrals over the orientation, not over the magnitude $k_{1}$ which is plotted on the abscissa, for $\eta=\eta^{\prime}=290 \mathrm{Mpc} / c$ and (from top to bottom in the second peak) $k=0.02 \mathrm{Mpc}^{-1}, k=0.04 \mathrm{Mpc}^{-1}, k=0.06 \mathrm{Mpc}^{-1}$ and $k=0.08 \mathrm{Mpc}^{-1}$. The primordial power-spectrum is set to $P_{\Phi}(k)=1 / k^{3}$. The main contribution is always located around $k_{1} \approx k$. If $k_{1}$ is larger than $k$, the integrand is suppressed by two primordial power spectra with large arguments. This allows us to cut the $k_{1}$ integration at some $k_{1}$ sufficiently larger than $k$. 


\begin{tabular}{|c|c|c|}
\hline$X$ & tight-coupling solution & numerical value \\
\hline \hline$I$ & $8063 \pm 9$ & $8128 \pm 10$ \\
\hline$E$ & 0 & $14.6 \pm 2.9$ \\
\hline$B$ & 0 & $0.01 \pm 0.02$ \\
\hline
\end{tabular}

Table 1: Comparison of the tight-coupling solution and the numerical result for the second-order power spectrum $P_{22,2}^{X(2)}(k) ; k=0.05 \mathrm{Mpc}^{-1}, \eta=200 \mathrm{Mpc} / c$, primordial power-spectrum $P_{\Phi}(k)=$ $1 / k^{3}$.

first order solutions up to $l=4$.

We compute all first-order quantities and Green functions in advance, before performing the integration. However, in the integrand they are required as function of conformal time and wave number. Thus we tabulate the solutions, storing their values for $n_{k}$ equidistant wave-number values $k_{0}=0, \ldots, k_{n_{k}-1}=k_{\max }$ and $n_{a}$ time values $\eta_{0}=\eta_{\text {ini }}, \ldots$, $\eta_{n_{a}-1}=\eta_{\mathrm{fs}}$. The time steps are chosen such that the corresponding scale factors are equidistant. We obtain one $n_{k} \times n_{a}$ array for each first order quantity and one $n_{k} \times n_{a} \times n_{a}$ array for each Green function. In the further computation, the tables are interpolated using basis splines (B-splines). To obtain accurate results, the distance between successive $\eta$ and $k$ values has to be much smaller than the typical scale of the interpolated function's fluctuations.

\subsection{Test in the tight-coupling regime}

In order to test our numerics we compare the numerical results for the two-point function at early times before recombination with the analytically known second-order tight-coupling solution. At early times polarization vanishes and the photon quadrupole is given by $10 C_{m_{1}, m}^{-, 2} v_{e,\left[m_{1}\right]}^{(1)}\left(\boldsymbol{k}_{1}\right) v_{e,\left[m_{2}\right]}^{(1)}\left(\boldsymbol{k}_{2}\right)$. Table 1 shows the analytical and numerical values of the tensor power-spectrum $P_{22,2}^{X(2)}(k)$ for $k=0.05 \mathrm{Mpc}^{-1}$ at conformal time $\eta=200 \mathrm{Mpc} / c$. For this test, the primordial power-spectrum is set to $P_{\Phi}(k)=1 / k^{3}$. As expected, the polarization modes are strongly suppressed and the unpolarized quadrupole has the correct size. The small difference between the numerical values and the tight-coupling solution comes from the finite scattering rate limiting the validity of the tight-coupling solution for which an infinite scattering rate is assumed. Evidently, in contrast to the unpolarized quadrupole, the E mode is strongly suppressed. Even stronger suppressed is the B mode since it is not coupled directly to the unsuppressed intensity quadrupole but only indirectly via the E mode by the free-streaming term.

\subsection{Numerical stability tests}

The numerical error of our final result is made up of a systematic error and the statistical error from the Monte Carlo integration. While for the latter the VEGAs integration routine provides a reliable estimate, quantifying the systematic error requires involved analysis.

The systematic error is controlled by five parameters: $\eta_{\mathrm{fs}}, k_{\max }, n_{a}, n_{k}$ and $n_{l}$. Larger values for any of the five parameters yield better results but require more CPU time. To balance both demands, we study the variation of the numerical result under variation 

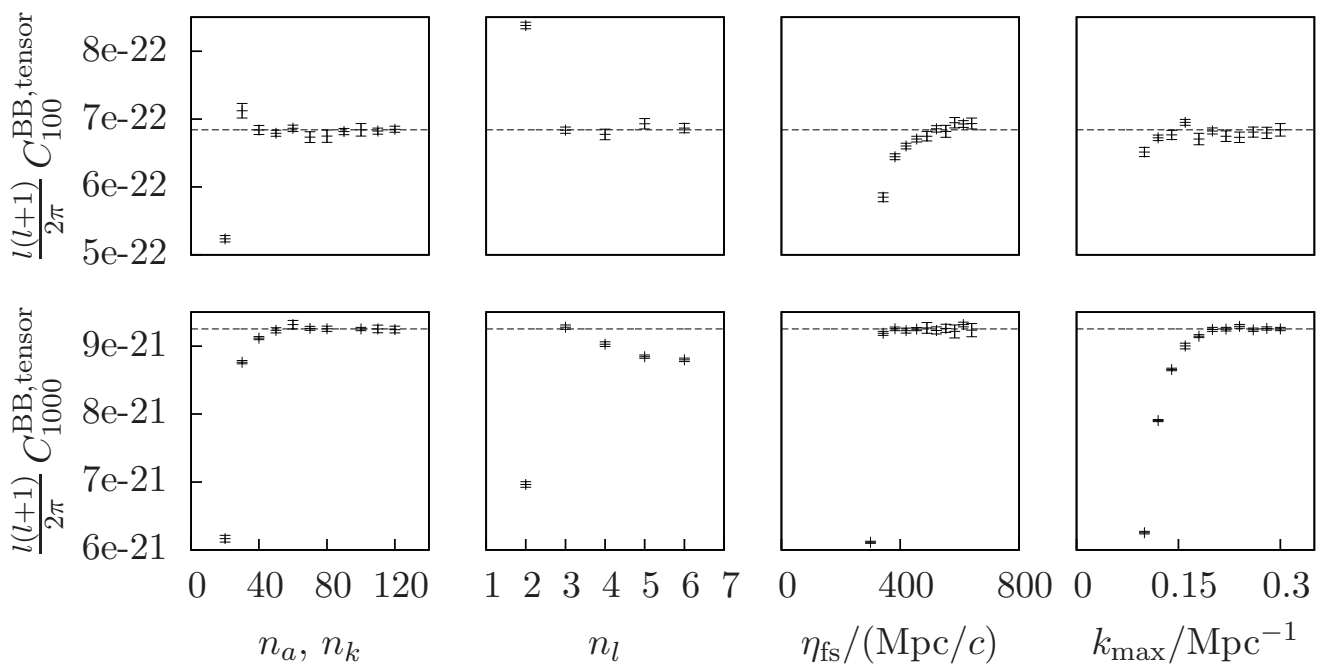

Figure 6: Dependence of the numerical result on the numerical parameters. The tensor $(m= \pm 2)$ contribution to the $C_{l}^{B B}$ value for $l=100$ (top row) and for $l=1000$ (bottom row) is plotted vs. (from left to right) the number of scale-factor steps $n_{a}$ and wave-number steps $n_{k}$, the number of multipole moments taken into account in the sources $S_{n}$, the scale factor $a_{\mathrm{fs}}$ from which we start using the free-streaming solution, and the truncation of the wave-number integrals $k_{\max }$. All parameters except for the parameter used as abscissa are kept fixed at the values $n_{a}=n_{k}=100$, $n_{l}=3, \eta_{\mathrm{fs}}=500 \mathrm{Mpc} / c$ (corresponding to $a_{\mathrm{fs}}=0.002064$ ) and $k_{\max }=0.3 \mathrm{Mpc}^{-1}$. The central value of the result obtained using the default parameters is indicated by dashed lines.

of the parameters. Values which yields a systematic error on the few percent level are $n_{a}=n_{k}=100, n_{l}=3, \eta_{\mathrm{fs}}=500 \mathrm{Mpc} / c$ and $k_{\max }=0.3 \mathrm{Mpc}^{-1}$.

Figure 6 shows the stability of the result for the tensor contribution $(m= \pm 2)$ to the angular power spectrum computed using these parameters. In the figure we identify the choice of $\eta_{\mathrm{fs}}$ to dominate the systematic error for small $l$, whereas for large $l$ neglecting higher multipoles in the sources is the most relevant approximation. This can be easily understood by considering the $l$ dependence of the spherical Bessel-function $j_{l}\left(k\left(\eta_{0}-\eta\right)\right)$ in the line-of-sight integral. For given $l$, the Bessel-function is small if $k\left(\eta_{0}-\eta\right) \lesssim l$ and oscillates for $k\left(\eta_{0}-\eta\right) \gtrsim l$; the main contribution to the integral comes from $k\left(\eta_{0}-\eta\right) \approx l$. Since $\eta_{0} \gg \eta_{\mathrm{fs}} \geq \eta$, the integral is therefore dominated by contributions at $k \approx l / \eta_{0}$. Accordingly, the precondition for free streaming, $|\dot{\kappa}| \ll k$, is fulfilled at later times for small $l$ than for large $l$. Consequently, the computation for small $l$ requires a large $\eta_{\mathrm{fs}}$. With increasing $l$, the main contribution to the integral moves to larger $k$ values. While for small $k$ the lowest multipole moments of the first-order result are largest, with increasing $k$ they are superseded by the higher moments. This can already be noticed in Fig. 6, where increasing the number of multipole moments in the source terms from $n_{l}=3$ to $n_{l}=4$ or $n_{l}=5$ changes the result significantly for $l=1000$, but not for $l=100$. However, Fig. 7 shows that for both, the tensor and the vector contribution to the $C_{l}^{B B}$ B-mode angular power spectrum this effect does not gain importance for even larger $l$ : the spectra for $n_{l}=3$ are close to the ones computed with $n_{l}=4$ and 5 for all $l$, whereas $n_{l}=2$ is clearly insufficient to cover the entire range up to $l \approx 2000$. 

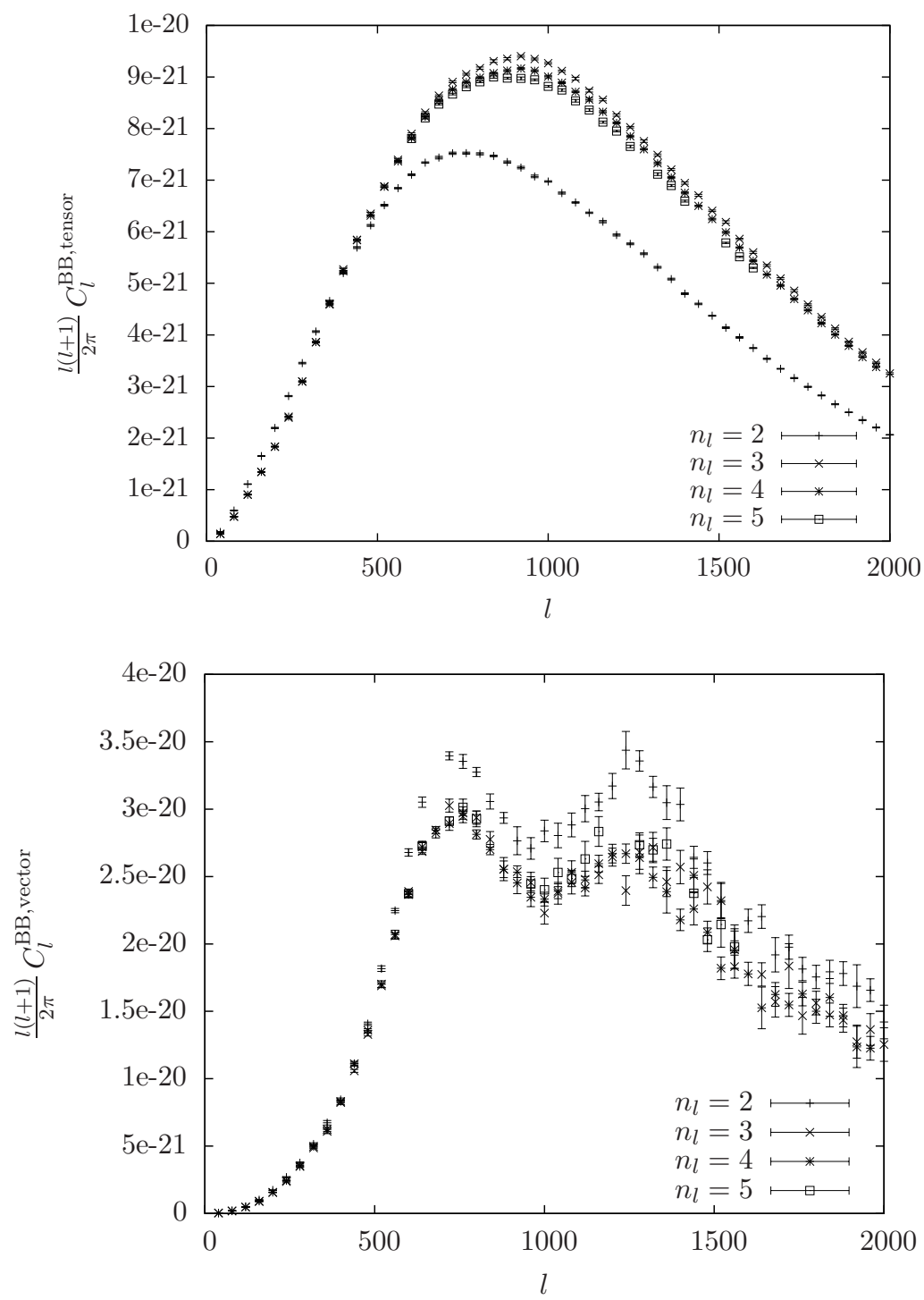

Figure 7: Tensor $\left(|m|=2\right.$, upper plot) and vector contribution $(|m|=1$, lower plot $)$ to the $C_{l}^{B B}$ spectrum, computed with different values of $n_{l}$, the number of multipoles in the source terms.

\section{Result and conclusion}

We summarize our results by showing the B-mode angular power spectrum $C_{l}^{B B}$ from the second-order collision term in Fig. 8 both, on a logarithmic (upper plot) and on a linear scale (lower plot). Similar to the weak-lensing induced contribution, the angular power spectrum grows rapidly with $l$ until it reaches a maximum around $l \approx 1000$. At $l \lesssim 200$ the tensor mode contribution $(|m|=2)$ is larger than the vector $(|m|=1)$ contribution, but the latter dominates in the peak region. The vector mode also shows a double peak structure around $l \approx 1000$.

We find that for $|m|=1$ the source terms $S_{n}$ are dominated by the terms containing 

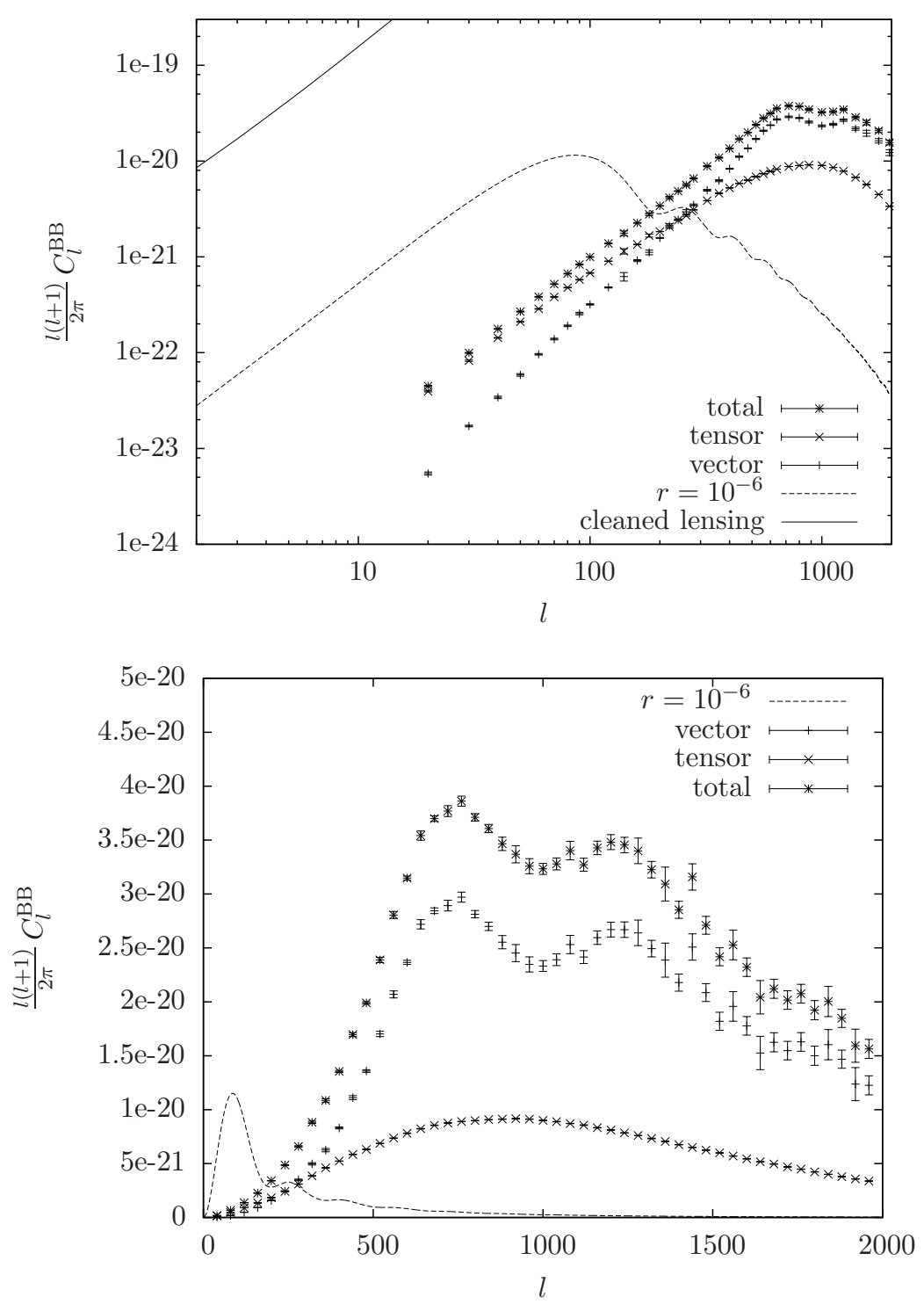

Figure 8: $\quad$ The $C_{l}^{B B}$ angular power spectrum from second-order scattering sources (points) compared to the spectrum induced by primordial tensor perturbations with tensor-to-scalar ratio $r=10^{-6}$ (dashed line) and the weak-lensing signal cleaned by a factor 40 (solid line, upper panel only). We display separately the second-order contributions from vector $(|m|=1)$ and tensor $(|m|=2)$ perturbations, and their sum. The upper and lower plot show the same numerical data, first on a logarithmic, then on a linear scale. (Numerical parameters are fixed to the values $n_{a}=n_{k}=100, n_{l}=4, \eta_{\mathrm{fs}}=500 \mathrm{Mpc} / c$, and $k_{\max }=0.3 \mathrm{Mpc}^{-1}$.)

a product of the first-order electron velocity $v_{e,[m]}^{(1)}$ and radiation dipole $\Delta_{I, 1 m}^{(1)}$ (closely related to the electron velocity in tight coupling) with each other or themselves. Setting all other first-order quantities in $S_{n}$ to zero, only a few terms remain. The source term $S_{I, l m}$ consists of only the fourth and seventh line of the right-hand side of Eq. (2.18), the source $S_{E, l m}$ of the third and fourth line of the right-hand side of Eq. (2.19), and the sources 
$S_{B, l m}$ and $S_{v_{e}, 1 m}$ vanish altogether as can be seen from Eq. (2.20) and Eq. (2.21). With these approximations, we obtain the double-peaked spectrum with the first and second peak reaching more than $80 \%$ and $60 \%$, respectively, of the corresponding peaks in the full result, Fig. 8. This indicates that the double-peak structure is related to the evolution of the first-order electron velocity/radiation dipole. The electron velocity oscillates over time until recombination and thus throughout the time interval relevant for the integration. The frequency depends on the wave number which in the source terms is $k_{1}$ and $k_{2}$, respectively. For the vector-mode contribution the wave-vector convolution integral is dominated by contributions at $k_{1} \approx k$ and $k_{2}$ small or vice versa and the $k$ integral by contributions at $k \approx l / \eta_{0}$. Since $\eta_{0} \approx 14000 \mathrm{Mpc} / c$, this relates the $l$ values of the two peaks, $l \approx 750$ and $l \approx 1250$, to the wave numbers $k \approx 0.05 \mathrm{Mpc}^{-1}$ and $k \approx 0.09 \mathrm{Mpc}^{-1}$. For both $k$ values, the oscillation of the electron velocity reaches an extremum at recombination, where for $k \approx 0.09 \mathrm{Mpc}^{-1}$ it has undergone one full oscillation more than for $k \approx 0.05 \mathrm{Mpc}^{-1}$. The intermediate wave number $k \approx 0.07 \mathrm{Mpc}^{-1}$, for which the oscillation stops at recombination with a phase shift of only half a period, corresponds to $l \approx 1000$. At this $l$ value we observe a local minimum of the spectrum. The tensor spectrum does not show a double-peak structure, since a larger range of wave numbers contributes to the convolution integral so that any oscillations are averaged out after integration.

The amplitude of collision-induced B-mode polarization is several orders of magnitude smaller than the weak-lensing signal. At $l \approx 200$ it is comparable to the B-mode power generated by primordial gravitational waves with a tensor-to-scalar ratio $r \approx 10^{-6}$ (shown as dashed line in the Figure), growing to $2 \cdot 10^{-4}$ at $l \approx 1000$. The small amplitude of the collisional contribution is presumably due to the fact that the sources involve only the cross-coupling of the first-order photon perturbations to the velocity of the baryon fluid and further are mainly localized in time to the recombination era. It may therefore be of interest to include the reionization history into the computation to obtain a realistic estimate of the power spectrum at low values $l \approx 10$.

The collision-induced spectrum may be compared to the spectrum induced by secondorder vector and tensor metric perturbations [10]. These act as sources for vector and tensor perturbations of the radiation intensity, which can then be converted into E-mode polarization by Thomson scattering through the first-order collision term, and subsequently to B-mode polarization by free-streaming. We find that both contributions, the present one and the one from metric perturbations, are similar in magnitude. If the weak-lensing signal could be "cleaned" completely, these second-order terms would constitute a background to the search for primordial gravitational waves at the level of $r<10^{-6} \ldots 2 \cdot 10^{-4}$ (depending on $l$ ), which represents a challenge to $\mathrm{CBR}$ polarization experiments.

\section{Acknowledgement}

We thank E. Dimastrogiovanni and C. Pitrou for helpful discussions and communications. This work is supported in part by the Gottfried Wilhelm Leibniz programme of the Deutsche Forschungsgemeinschaft (DFG) and the DFG Graduiertenkolleg "Elementarteilchenphysik an der TeV-Skala". 


\section{A. Summary of equations}

In this appendix we summarize further equations related to the content of the main text. For conventions and notation not stated explicitly we refer to Ref. [11]. The equations are given in Fourier space conjugate to position, vectors and tensors are decomposed into spherical components. Phase space densities are expanded into multipoles with respect to the photon momentum direction.

In the absence of first-order vector and tensor perturbations the first-order Boltzmann hierarchy for the energy-integrated photon phase space density multipoles (2.3) is given in conformal Newtonian gauge by

$$
\begin{aligned}
& \frac{\partial}{\partial \eta} \Delta_{I, l m}^{(1)}(\boldsymbol{k})+\sum_{ \pm}(\mp i) \Delta_{I,(l \pm 1) m_{1}}^{(1)}(\boldsymbol{k}) k^{\left[m_{2}\right]} C_{m_{1} m}^{ \pm, l}+4 \delta_{l 0} \dot{D}^{(1)}(\boldsymbol{k})+4 \delta_{l 1} i k^{[m]} A^{(1)}(\boldsymbol{k}) \\
& \quad=|\dot{\kappa}|\left\{-\Delta_{I, l m}^{(1)}(\boldsymbol{k})+\delta_{l 0} \Delta_{I, 00}^{(1)}(\boldsymbol{k})+4 \delta_{l 1} v_{e,[m]}^{(1)}(\boldsymbol{k})+\delta_{l 2} \frac{1}{10}\left(\Delta_{I, 2 m}^{(1)}(\boldsymbol{k})-\sqrt{6} \Delta_{E, 2 m}^{(1)}(\boldsymbol{k})\right)\right\} \\
& \frac{\partial}{\partial \eta} \Delta_{E, l m}^{(1)}(\boldsymbol{k})+\sum_{ \pm}(\mp i) \Delta_{E,(l \pm 1) m_{1}}^{(1)}(\boldsymbol{k}) k^{\left[m_{2}\right]} D_{m_{1} m}^{ \pm, l}-i \Delta_{B, l m_{1}}^{(1)}(\boldsymbol{k}) k^{\left[m_{2}\right]} D_{m_{1} m}^{0, l} \\
& \quad=|\dot{\kappa}|\left\{-\Delta_{E, l m}^{(1)}(\boldsymbol{k})-\delta_{l 2} \frac{\sqrt{6}}{10}\left(\Delta_{I, 2 m}^{(1)}(\boldsymbol{k})-\sqrt{6} \Delta_{E, 2 m}^{(1)}(\boldsymbol{k})\right)\right\}, \\
& \frac{\partial}{\partial \eta} \Delta_{B, l m}^{(1)}(\boldsymbol{k})+\sum_{ \pm}(\mp i) \Delta_{B,(l \pm 1) m_{1}}^{(1)}(\boldsymbol{k}) k^{\left[m_{2}\right]} D_{m_{1} m}^{ \pm, l}+i \Delta_{E, l m_{1}}^{(1)}(\boldsymbol{k}) k^{\left[m_{2}\right]} D_{m_{1} m}^{0, l} \\
& \quad=|\dot{\kappa}|\left\{-\Delta_{B, l m}^{(1)}(\boldsymbol{k})\right\} .
\end{aligned}
$$

Since B-mode polarization is not generated at first order from scalar perturbations, we may set $\Delta_{B, l m}^{(1)}(\boldsymbol{k})=0$ in these equations. The Boltzmann equations for the massless neutrino variables $\Delta_{\nu, l m}^{(1)}(\boldsymbol{k})$ are the same as the equation for $\Delta_{I, l m}^{(1)}(\boldsymbol{k})$ with the collision term on the right-hand side set to zero. Baryons and electrons form a tightly coupled fluid with first-order variables $\delta_{b}^{(1)}(\boldsymbol{k})=\left[\delta \rho_{b} / \bar{\rho}_{b}\right]^{(1)}(\boldsymbol{k}), v_{e,[m]}^{(1)}(\boldsymbol{k})$ determined by

$$
\begin{aligned}
& \frac{\partial}{\partial \eta} \delta_{b}^{(1)}(\boldsymbol{k})-\sum_{m=-1}^{1}(-1)^{m} i k^{[m]} v_{e,[-m]}^{(1)}(\boldsymbol{k})+3 \dot{D}^{(1)}(\boldsymbol{k})=0, \\
& \left(\frac{\partial}{\partial \eta}+H_{C}\right) v_{e,[m]}^{(1)}(\boldsymbol{k})+i k^{[m]} A^{(1)}(\boldsymbol{k})=-\frac{|\dot{\kappa}|}{4 R}\left\{4 v_{e,[m]}^{(1)}(\boldsymbol{k})-\Delta_{I, 1 m}^{(1)}(\boldsymbol{k})\right\}
\end{aligned}
$$

with $R=3 \bar{\rho}_{b} /\left(4 \bar{\rho}_{\gamma}\right)$. The cold dark matter perturbations $\delta_{c}^{(1)}(\boldsymbol{k})=\left[\delta \rho_{c} / \bar{\rho}_{c}\right]^{(1)}(\boldsymbol{k}), v_{c,[m]}^{(1)}(\boldsymbol{k})$ are described by the same equations with the collision term on the right-hand side set to zero. The system closes with the Einstein equations for the first-order metric perturbations, which can be put into the form

$$
\begin{aligned}
& k^{2} D^{(1)}(\boldsymbol{k})+3 H_{C} \dot{D}^{(1)}(\boldsymbol{k})-3 H_{C}^{2} A^{(1)}(\boldsymbol{k})=4 \pi G a^{2} \delta \rho^{(1)}(\boldsymbol{k}), \\
& C_{m_{1} m}^{-, 2} k_{\left[m_{1}\right]} k_{\left[m_{2}\right]}\left(A^{(1)}+D^{(1)}\right)(\boldsymbol{k})=8 \pi G a^{2} \alpha_{m} \Sigma_{[m]}^{(1)}(\boldsymbol{k}) .
\end{aligned}
$$


The density perturbation and anisotropic stress are given by

$$
\begin{aligned}
& \delta \rho^{(1)}(\boldsymbol{k})=\bar{\rho}_{b} \delta_{b}^{(1)}+\bar{\rho}_{c} \delta_{c}^{(1)}+\bar{\rho}_{\gamma} \Delta_{I, 00}^{(1)}+\bar{\rho}_{\nu} \Delta_{\nu, 00}^{(1)}, \\
& \Sigma_{[m]}^{(1)}(\boldsymbol{k})=\frac{2}{15 \alpha_{m}}\left(\bar{\rho}_{\gamma} \Delta_{I, 2 m}^{(1)}+\bar{\rho}_{\nu} \Delta_{\nu, 2 m}^{(1)}\right) .
\end{aligned}
$$

In practice, we solve the first-order equations only for mode vectors $\boldsymbol{k}$ aligned with the 3 -axis, in which case $k^{[0]}=i k$ and $k^{[ \pm 1]}=0$. In the absence of vector and tensor modes the $m= \pm 1, \pm 2$ components all vanish for this choice of $\boldsymbol{k}$. The solution for general $\boldsymbol{k}$ needed in the source term of the second-order equations is obtained from the $m=0$ solution for $\boldsymbol{k}=k \boldsymbol{e}_{3}$ by a rotation. In general, if $T_{l m}(\boldsymbol{k})$ is a spherical tensor of rank $l$, the relation between its components for $\boldsymbol{k}=k \hat{\boldsymbol{k}}$ and $\boldsymbol{k}=k \boldsymbol{e}_{3}$ is

$$
T_{l m}(\boldsymbol{k})=\sum_{m^{\prime}} T_{l m^{\prime}}\left(k \boldsymbol{e}_{3}\right) D_{m^{\prime} m}^{(l)}\left(R^{-1}\right)=\sqrt{\frac{4 \pi}{2 l+1}} \sum_{m^{\prime}} T_{l m^{\prime}}\left(k \boldsymbol{e}_{3}\right) Y_{l m}^{-m^{\prime}}(\hat{\boldsymbol{k}})
$$

where $D_{m^{\prime} m}^{(l)}\left(R^{-1}\right)_{m^{\prime} m}$ denotes the Wigner function for the rotation $R^{-1}$ with $\hat{\boldsymbol{k}}=R \boldsymbol{e}_{3}$, which can be expressed in terms of the spin-weighted spherical harmonics [21]. We apply this to $A^{(1)}, D^{(1)}, \Delta_{I, 00}^{(1)}, \delta_{b}^{(1)}$ etc. with $l=0$, to $\Delta_{I, 1 m}^{(1)}, v_{e,[m]}^{(1)}$ etc. with $l=1$ and to $\Delta_{X, l m}^{(1)}$ and $\Delta_{\nu, l m}^{(1)}$ with any $l$. In this case, due to the absence of first-order vector and tensor perturbations, only the $m^{\prime}=0$ term contributes to the sum in Eq. (A.5).

Since we do not consider polarization induced by the second-order vector and tensor metric perturbations, themselves induced by the first-order scalar modes, we do not need to solve the second-order Einstein equations. The only second-order quantity other than the photon perturbations that we must solve for is the electron velocity for which we use the truncated equation (2.21) as explained in the main text. For completeness we provide here the complete equations for the second-order variables of the baryon-electron fluid. We define the fluid variables through the energy-momentum tensor in the local inertial frame at rest and aligned with the general coordinate system

$$
\begin{aligned}
T_{A B} & =\left[e_{A}\right]^{\mu}\left[e_{B}\right]^{\nu} T_{\mu \nu}=(\rho+p) u_{A} u_{B}-p \eta_{A B}+\Sigma_{A B} \\
& =\int \frac{d^{3} \boldsymbol{p}}{(2 \pi)^{3}} g(\eta, \boldsymbol{x}, \boldsymbol{p}) \frac{p_{A} p_{B}}{E} .
\end{aligned}
$$

Here $p^{A}$ denotes the physical (not co-moving) particle momentum in the local frame and $g(\eta, \boldsymbol{x}, \boldsymbol{p})$ the phase-space distribution, for which we take a local Maxwell-Boltzmann distribution. The four-velocity is parameterized in the form $u^{A}=\left(1 / \sqrt{1-\boldsymbol{v}^{2}}, \boldsymbol{v}\right)$. We then calculate the conformal-time derivative of the expression in the second line using the results of Ref. [11]. Together with

$$
T_{00}=\rho+\bar{\rho}\left[\boldsymbol{v}^{(1)}\right]^{2}+\ldots, \quad T_{0 i}=-\rho v^{i}+\ldots,
$$


valid to second order in perturbations, we obtain the desired equations of the density and velocity perturbation of the baryon-electron fluid to second order:

$$
\begin{aligned}
& \frac{\partial}{\partial \eta} \delta_{b}^{(2)}(\boldsymbol{k})-\sum_{m=-1}^{1}(-1)^{m} i k^{[m]} v_{e,[-m]}^{(2)}(\boldsymbol{k})+3 \dot{D}^{(2)}(\boldsymbol{k}) \\
& -\sum_{m=-1}^{1}(-1)^{m}\left(i k^{[m]} \delta_{b}^{(1)}+i k_{2}^{[m]}\left(A^{(1)}-D^{(1)}\right)+2 i k_{1}^{[m]} D^{(1)}\right)\left(\boldsymbol{k}_{1}\right) v_{e,[-m]}^{(1)}\left(\boldsymbol{k}_{2}\right) \\
& +\sum_{m=-1}^{1}(-1)^{m} H_{C} v_{e,[m]}^{(1)}\left(\boldsymbol{k}_{1}\right) v_{e,[-m]}^{(1)}\left(\boldsymbol{k}_{2}\right)-6 D^{(1)}\left(\boldsymbol{k}_{1}\right) \dot{D}^{(1)}\left(\boldsymbol{k}_{2}\right)+3 \dot{D}^{(1)}\left(\boldsymbol{k}_{1}\right) \delta_{b}^{(1)}\left(\boldsymbol{k}_{2}\right) \\
& =-\frac{|\dot{\kappa}|}{4 R} \sum_{m=-1}^{1}(-1)^{m} v_{e,[-m]}^{(1)}\left(\boldsymbol{k}_{1}\right)\left(4 v_{e,[m]}^{(1)}-\Delta_{I, 1 m}^{(1)}\right)\left(\boldsymbol{k}_{2}\right), \\
& \left(\frac{\partial}{\partial \eta}+H_{C}\right)\left(v_{e,[m]}^{(2)}-B_{[m]}^{(2)}\right)(\boldsymbol{k})+i k^{[m]} A^{(2)}(\boldsymbol{k}) \\
& -i k_{1}^{[m]} A^{(1)}\left(\boldsymbol{k}_{1}\right)\left(A^{(1)}+D^{(1)}\right)\left(\boldsymbol{k}_{2}\right)+\dot{D}^{(1)}\left(\boldsymbol{k}_{1}\right) v_{e,[m]}^{(1)}\left(\boldsymbol{k}_{2}\right) \\
& -\sum_{m^{\prime}=-1}^{1}(-1)^{m^{\prime}} i k_{2}^{\left[-m^{\prime}\right]} v_{e,\left[m^{\prime}\right]}^{(1)}\left(\boldsymbol{k}_{1}\right) v_{e,[m]}^{(1)}\left(\boldsymbol{k}_{2}\right) \\
& =-\frac{|\dot{\kappa}|}{4 R}\left\{4 v_{e,[m]}^{(2)}(\boldsymbol{k})-\Delta_{I, 1 m}^{(2)}(\boldsymbol{k})+\left(A^{(1)}+\left[\frac{\delta x_{e}}{x_{e}}\right]^{(1)}\right)\left(\boldsymbol{k}_{1}\right)\left(4 v_{e,[m]}^{(1)}\left(\boldsymbol{k}_{2}\right)-\Delta_{I, 1 m}^{(1)}\left(\boldsymbol{k}_{2}\right)\right)\right. \\
& \left.-v_{e,\left[m_{2}\right]}^{(1)}\left(\boldsymbol{k}_{1}\right) \Delta_{I, 2 m_{1}}^{(1)}\left(\boldsymbol{k}_{2}\right) C_{m_{1} m}^{+, 1}+4 v_{e,[m]}^{(1)}\left(\boldsymbol{k}_{1}\right) \Delta_{I, 00}^{(1)}\left(\boldsymbol{k}_{2}\right)\right\} \text {. }
\end{aligned}
$$

The left-hand sides of these equations are obtained from the $l=0$ and $l=1$ moments of the Boltzmann equation for massive particles given in [11]. The right-hand sides follow from the collision term for photons using the tight coupling of baryons and electrons through Coulomb scattering and energy-momentum conservation in photon-electron Compton scattering. Eqs. (A.8) and (A.9) agree with the corresponding results in Ref. [22].

Finally, the coupling coefficients $C_{m_{1} m_{2}}^{ \pm, l}$ and $D_{m_{1} m_{2}}^{0, l}$ that appear in the Boltzmann equations read

$$
\begin{aligned}
C_{m \pm 1, m}^{+, l} & =-\frac{\sqrt{(l+1 \pm m)(l+2 \pm m)}}{\sqrt{2}(2 l+3)} \\
C_{m, m}^{+, l} & =\frac{\sqrt{(l+1)^{2}-m^{2}}}{2 l+3} \\
C_{m \pm 1, m}^{-, l} & =\frac{\sqrt{(l-1 \mp m)(l \mp m)}}{\sqrt{2}(2 l-1)} \\
C_{m, m}^{-, l} & =\frac{\sqrt{l^{2}-m^{2}}}{2 l-1}
\end{aligned}
$$




$$
\begin{aligned}
D_{m \pm 1, m}^{0, l} & =\mp \frac{\sqrt{2(l+1 \pm m)(l \mp m)}}{l(l+1)} \\
D_{m, m}^{0, l} & =-\frac{2 m}{l(l+1)} .
\end{aligned}
$$

\section{B. Derivation of the line-of-sight solution}

The line-of-sight solution of the first-order Boltzmann equations have been derived in Ref. [14] (see also Ref. [15]). We provide here a generalization which allows for more general source terms including higher multipole moments and polarization modes.

Our aim is to solve the equation

$$
\dot{\Delta}_{n}+k C_{n m} \Delta_{m}=-|\dot{\kappa}| \Delta_{n}+\rho_{n}
$$

for $\boldsymbol{k}=\boldsymbol{k}_{3}$. First we solve the homogeneous differential equation without the source term $\rho_{n}$. This can be done easily after reversing the multipole decomposition. Then the homogeneous equation takes the simple form:

$$
\dot{\Delta}_{a b}+i \boldsymbol{n} \cdot \boldsymbol{k} \Delta_{a b}=-|\dot{\kappa}| \Delta_{a b},
$$

where $a b=++,+-,-+,--$ are the polarization indices. This equation is solved by

$$
\Delta_{a b}(\eta)=e^{-i \boldsymbol{n} \cdot \boldsymbol{k}\left(\eta-\eta^{\prime}\right)-\kappa\left(\eta, \eta^{\prime}\right)} \Delta_{a b}\left(\eta^{\prime}\right),
$$

where $\kappa\left(\eta, \eta^{\prime}\right)$ is the integral over $|\dot{\kappa}|$ from $\eta^{\prime}$ to $\eta$. If the first argument of $\kappa$ is the conformal time today $\eta_{0}$, we omit this argument in the following and in the main text, and write $\kappa(\eta)=\kappa\left(\eta_{0}, \eta\right)$. Next, we decompose this solution into multipoles. For this purpose, we expand the exponential in spherical harmonics and $\Delta_{a b}\left(\eta^{\prime}\right)$ in spin-weighted spherical harmonics,

$$
\begin{aligned}
\Delta_{a b}(\eta)= & \sum_{l_{1}} e^{-\kappa\left(\eta, \eta^{\prime}\right)}(-i)^{l_{1}} \sqrt{4 \pi\left(2 l_{1}+1\right)} j_{l_{1}}\left(k\left(\eta-\eta^{\prime}\right)\right) Y_{l_{1} 0}(\boldsymbol{n}) \\
& \times \sum_{l_{2}, m} i^{-l_{2}} \sqrt{\frac{4 \pi}{2 l_{2}+1}} \Delta_{a b, l_{2} m}\left(\eta^{\prime}\right) Y_{l_{2} m}^{s}(\boldsymbol{n})
\end{aligned}
$$

and apply on both sides $i^{l} \sqrt{\frac{2 l+1}{4 \pi}} \int d \Omega Y_{l m}^{s *}(\boldsymbol{n})$ to obtain

$$
\begin{aligned}
\Delta_{a b, l m}(\eta)= & \sum_{l_{1}, l_{2}} e^{-\kappa\left(\eta, \eta^{\prime}\right)} i^{l-l_{1}-l_{2}} \frac{(2 l+1)\left(2 l_{1}+1\right)}{2 l_{2}+1} j_{l_{1}}\left(k\left(\eta-\eta^{\prime}\right)\right) \\
& \times\left(\begin{array}{ccc}
l & l_{1} & l_{2} \\
m & 0 & m
\end{array}\right)\left(\begin{array}{ccc}
l & l_{1} & l_{2} \\
-s & 0 & -s
\end{array}\right) \Delta_{a b, l_{2} m}\left(\eta^{\prime}\right) .
\end{aligned}
$$

The spin $s$ is zero for $a b=++,--$ and \pm 2 for $a b= \pm \mp$. Finally, instead of the photon helicity state densities, we use the Stokes parameter distributions

$$
\begin{aligned}
\Delta_{I, l m} & =\frac{1}{2}\left(\Delta_{++, l m}+\Delta_{--, l m}\right), \\
\Delta_{E, l m} & =\frac{1}{2}\left(\Delta_{+-, l m}+\Delta_{-+, l m}\right), \\
\Delta_{B, l m} & =\frac{i}{2}\left(\Delta_{+-, l m}-\Delta_{-+, l m}\right) .
\end{aligned}
$$


On the right-hand side of Eq. (B.7) and Eq. (B.8) there is a sum and a difference, respectively, of Clebsch-Gordan coefficients with opposite spin. They are related by

$$
\left(\begin{array}{ccc}
l & l_{1} & l_{2} \\
-s & 0 & -s
\end{array}\right)=(-1)^{l-l_{1}-l_{2}}\left(\begin{array}{ccc}
l & l_{1} & l_{2} \\
s & 0 & s
\end{array}\right) \text {. }
$$

Depending on the parity of $l-l_{1}-l_{2}$ we obtain either a sum or a difference of the +and -+ components. For odd parity, this leads to a mixing of $\mathrm{E}$ and $\mathrm{B}$ modes. The resulting mode coupling can be written in a compact form as matrix $H_{X X^{\prime}}$ defined by $H_{X X^{\prime}}($ even $)=\delta_{X X^{\prime}}$ and $H_{X X^{\prime}}($ odd $)=\delta_{X I} \delta_{X^{\prime} I}+i \delta_{X E} \delta_{X^{\prime} B}-i \delta_{X B} \delta_{X^{\prime} E}$. Then

$$
\begin{aligned}
\Delta_{X, l m}(\eta)= & \sum_{l_{1}, l_{2}} \sum_{X^{\prime}=I, E, B} e^{-\kappa\left(\eta, \eta^{\prime}\right)} i^{l-l_{1}-l_{2}} \frac{(2 l+1)\left(2 l_{1}+1\right)}{2 l_{2}+1} j_{l_{1}}\left(k\left(\eta-\eta^{\prime}\right)\right) \\
& \times\left(\begin{array}{ccc}
l & l_{1} & l_{2} \\
m & 0 & m
\end{array}\right)\left(\begin{array}{ccc}
l & l_{1} & l_{2} \\
F_{X} & 0 & F_{X^{\prime}}
\end{array}\right) H_{X X^{\prime}}^{*}\left(l-l_{1}-l_{2}\right) \Delta_{X^{\prime}, l_{2} m}\left(\eta^{\prime}\right),
\end{aligned}
$$

where $F_{I}=0, F_{E}=F_{B}=-2$. A more detailed explanation of the effect which introduces the matrix $H$ and the properties of spin-weighted spherical harmonics can be found in Ref. [11]. With the solution of the homogeneous equation at hand, we can immediately write down the solution of the inhomogeneous equation:

$$
\begin{aligned}
\Delta_{X, l m}(\eta)= & \int d \eta^{\prime} \sum_{l_{1}, l_{2}} \sum_{X^{\prime}=I, E, B} e^{-\kappa\left(\eta, \eta^{\prime}\right)} i^{l-l_{1}-l_{2}} \frac{(2 l+1)\left(2 l_{1}+1\right)}{2 l_{2}+1} j_{l_{1}}\left(k\left(\eta-\eta^{\prime}\right)\right) \\
& \times\left(\begin{array}{ccc}
l & l_{1} & l_{2} \\
m & 0 & m
\end{array}\right)\left(\begin{array}{ccc}
l & l_{1} & l_{2} \\
F_{X} & 0 & F_{X^{\prime}}
\end{array}\right) H_{X X^{\prime}}^{*}\left(l-l_{1}-l_{2}\right) \rho_{X^{\prime}, l_{2} m}\left(\eta^{\prime}\right) .
\end{aligned}
$$

Using the multi-index notation from the main text, we can define the function

$$
\begin{aligned}
j_{n m}(x)= & \sum_{l_{1}} i^{l_{n}-l_{1}-l_{m}} \frac{\left(2 l_{n}+1\right)\left(2 l_{1}+1\right)}{2 l_{m}+1} j_{l_{1}}(x) H_{X_{n} X_{m}}^{*}\left(l_{n}-l_{1}-l_{m}\right) \\
& \times\left(\begin{array}{ccc}
l_{n} & l_{1} & l_{m} \\
m_{n} & 0 & m_{m}
\end{array}\right)\left(\begin{array}{ccc}
l_{n} & l_{1} & l_{m} \\
F_{X_{n}} & 0 & F_{X_{m}}
\end{array}\right)
\end{aligned}
$$

and $\rho_{\left(v_{e}, 1 m\right)} \equiv 0$ to obtain the compact equation

$$
\Delta_{n}\left(\eta_{0}\right)=\int_{0}^{\eta_{0}} d \eta e^{-\kappa(\eta)} j_{n m}\left(k\left(\eta_{0}-\eta\right)\right) \rho_{m}(\eta)
$$

which holds for $X_{n}=I, E, B$.

\section{References}

[1] M. Kamionkowski, A. Kosowsky, A. Stebbins, A Probe of primordial gravity waves and vorticity, Phys. Rev. Lett. 78 (1997) 2058, astro-ph/9609132.

[2] U. Seljak, M. Zaldarriaga, Signature of gravity waves in polarization of the microwave background, Phys. Rev. Lett. 78 (1997) 2054, astro-ph/9609169. 
[3] J. Kovac et al., Detection of polarization in the cosmic microwave background using DASI, Nature 420 (2002) 772, astro-ph/0209478.

[4] C. L. Bennett et al. [ WMAP Collaboration ], First year Wilkinson Microwave Anisotropy Probe (WMAP) observations: Preliminary maps and basic results, Astrophys. J. Suppl. 148 (2003) 1, astro-ph/0302207.

[5] D. Larson et al., Seven-Year Wilkinson Microwave Anisotropy Probe (WMAP) Observations: Power Spectra and WMAP-Derived Parameters, arXiv:1001.4635 [astro-ph.CO].

[6] E. Komatsu et al., Seven-Year Wilkinson Microwave Anisotropy Probe (WMAP) Observations: Cosmological Interpretation, arXiv:1001.4538 [astro-ph.CO].

[7] A. Lewis and A. Challinor, Weak Gravitational Lensing of the CMB, Phys. Rept. 429 (2006) 1, astro-ph/0601594.

[8] M. Zaldarriaga and U. Seljak, Gravitational Lensing Effect on Cosmic Microwave Background Polarization, Phys. Rev. D 58 (1998) 023003, astro-ph/9803150.

[9] W. Hu and A. Cooray, Gravitational time delay effects on cosmic microwave background anisotropies, Phys. Rev. D 63 (2001) 023504, astro-ph/0008001.

[10] S. Mollerach, D. Harari, S. Matarrese, CMB polarization from secondary vector and tensor modes, Phys. Rev. D69 (2004) 063002, astro-ph/0310711.

[11] M. Beneke, C. Fidler, Boltzmann hierarchy for the cosmic microwave background at second order including photon polarization, Phys. Rev. D82 (2010) 063509, arXiv:1003.1834 [astro-ph.CO].

[12] C. Pitrou, The Radiative transfer at second order: A Full treatment of the Boltzmann equation with polarization, Class. Quant. Grav. 26 (2009) 065006, arXiv:0809.3036 [gr-qc].

[13] N. Bartolo, S. Matarrese, A. Riotto, CMB Anisotropies at Second-Order. 2. Analytical Approach, JCAP 0701 (2007) 019, astro-ph/0610110.

[14] U. Seljak, M. Zaldarriaga, A Line of sight integration approach to cosmic microwave background anisotropies,” Astrophys. J. 469 (1996) 437-444, astro-ph/9603033.

[15] W. Hu, M. J. White, CMB anisotropies: Total angular momentum method, Phys. Rev. D56 (1997) 596-615, astro-ph/9702170.

[16] S. Seager, D. D. Sasselov, D. Scott, A new calculation of the recombination epoch, Astrophys. J. 523 (1999) L1-L5, astro-ph/9909275.

[17] M. Galassi et al GNU Scientific Library Reference Manual (3rd Ed.) ISBN 0954612078 http://www.gnu.org/software/gsl/

[18] A. Lewis, A. Challinor, A. Lasenby, Efficient computation of CMB anisotropies in closed FRW models, Astrophys. J. 538 (2000) 473-476, astro-ph/9911177.

[19] M. Doran, Cmbeasy... an object oriented code for the cosmic microwave background, JCAP 0510 (2005) 011, astro-ph/0302138.

[20] G. P. Lepage, A New Algorithm for Adaptive Multidimensional Integration, J. Comput. Phys. 27 (1978) 192.

[21] J. N. Goldberg et al., Spin-s Spherical Harmonics and $\bar{\delta}$, J. Math. Phys. 8 (1967) 2155.

[22] C. Pitrou, J. -P. Uzan, F. Bernardeau, The cosmic microwave background bispectrum from the non-linear evolution of the cosmological perturbations, JCAP 1007 (2010) 003, arXiv:1003.0481 [astro-ph.CO]. 